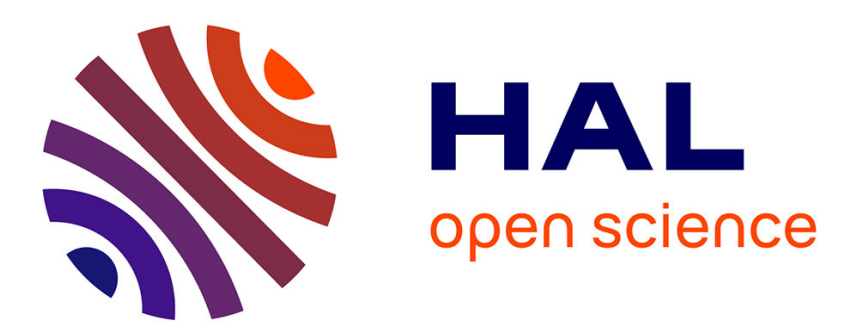

\title{
Effect of kinematic hardening on localized necking in substrate-supported metal layers
}

\author{
Mohamed Ben Bettaieb, Farid Abed-Meraim
}

\section{To cite this version:}

Mohamed Ben Bettaieb, Farid Abed-Meraim. Effect of kinematic hardening on localized necking in substrate-supported metal layers. International Journal of Mechanical Sciences, 2017, 123, pp.177-197. 10.1016/j.ijmecsci.2016.12.002 . hal-01541500

\section{HAL Id: hal-01541500 \\ https://hal.science/hal-01541500}

Submitted on $10 \mathrm{Jul} 2017$

HAL is a multi-disciplinary open access archive for the deposit and dissemination of scientific research documents, whether they are published or not. The documents may come from teaching and research institutions in France or abroad, or from public or private research centers.
L'archive ouverte pluridisciplinaire HAL, est destinée au dépôt et à la diffusion de documents scientifiques de niveau recherche, publiés ou non, émanant des établissements d'enseignement et de recherche français ou étrangers, des laboratoires publics ou privés. 


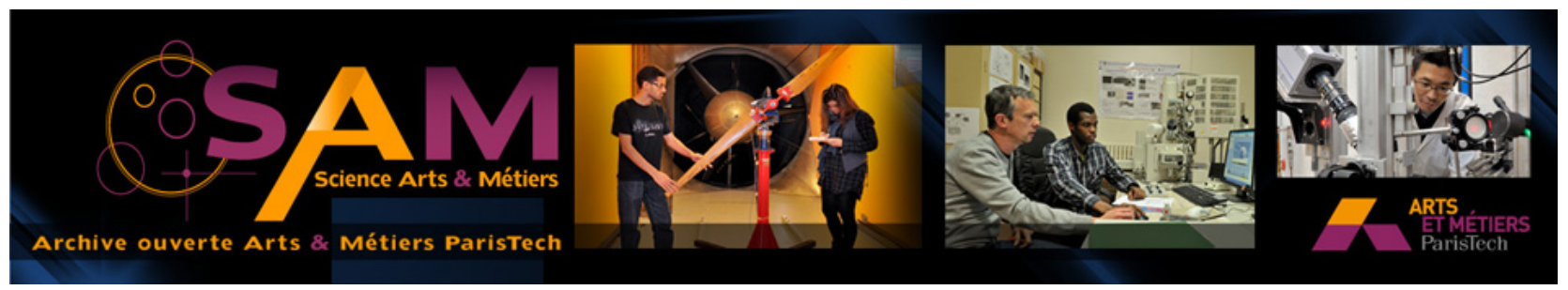

Science Arts \& Métiers (SAM)

is an open access repository that collects the work of Arts et Métiers ParisTech researchers and makes it freely available over the web where possible.

This is an author-deposited version published in: http://sam.ensam.eu

Handle ID: .http://hdl.handle.net/null

\section{To cite this version :}

Mohamed BEN BETTAIEB, Farid ABED-MERAIM - Effect of kinematic hardening on localized necking in substrate-supported metal layers - Effect of kinematic hardening on localized necking in substrate-supported metal layers - Vol. 123, p.177-197 - 2017 


\title{
Effect of kinematic hardening on localized necking in substrate-supported metal layers
}

\author{
Mohamed Ben Bettaieb $^{\mathrm{a}, \mathrm{b}}$, Farid Abed-Meraim ${ }^{\mathrm{a}, \mathrm{b}, *}$ \\ a LEM3, UMR CNRS 7239 Arts et Métiers ParisTech, 4 rue Augustin Fresnel, 57078 Metz Cedex 3, France \\ b DAMAS, Laboratory of Excellence on Design of Alloy Metals for low-mAss Structures, Université de Lorraine, France
}

\begin{abstract}
A B S T R A C T
Prediction of necking limits in thin substrate-supported metal layers, which are typically used as functional components in electronic devices, represents nowadays an ambitious challenge. The specific purpose of the current work is, first, to numerically investigate the effect of kinematic hardening on localized necking in a freestanding metal layer. Second, the impact of adding a substrate layer on the ductility evolution of the resulting elastomer/metal bilayer will be analyzed. The materials in the metal and substrate layers are assumed to be isotropic, incompressible and strain-rate independent. The behavior of the metal layer is described by a rigid-plastic model with mixed (isotropic and kinematic) hardening. The isotropic hardening contribution is modeled by the Hollomon law, while kinematic hardening is modeled by the Armstrong-Frederick law. The substrate layer is made of elastomer material whose mechanical behavior is assumed to be hyperelastic and modeled by a neo-Hookean constitutive law. The Marciniak-Kuczynski imperfection analysis is used to predict plastic flow localization. Through various numerical simulations, the influence of kinematic hardening on localized necking as well as the impact of the addition of an elastomer layer are specifically emphasized. Comparisons with experimental results are also carried out to assess the relevance of incorporating kinematic hardening in the constitutive modeling of freestanding metal sheets.
\end{abstract}

\section{Introduction}

Sheet metal forming processes are often limited by the initiation and development of localized necking due to the low ductility of the used materials. Such localized necking sets the maximum allowable straining that a sheet metal can undergo during metal forming processes, since this phenomenon is often precursor to material failure. Therefore, the development of new strategies that allow retarding the occurrence of necking is of significant practical interest. In the field of modern technologies, and especially functional components (e.g., flexible electronic devices), this aim is targeted by bonding ductile substrates (such as elastomer layers) to metal layers. Indeed, supporting a metal layer by a ductile substrate has proven to significantly improve the ductility of the resulting bilayer, as demonstrated by several authors [1-7]. In an industrial context, substrate-supported metal layers are being used in a variety of flexible electronic devices such as conductors and interconnects [8-11]. Despite the increasing industrial use of elastomer/metal bilayers, there is still a need for further studies for an in-depth understanding of the corresponding strain localization conditions. In the current paper, the objective is to numerically predict the onset of localized necking in a metal layer supported by an elastomer substrate. This will be illustrated through the concept of forming limit diagram (FLD), which is probably the most common representation of necking limits, as initially introduced by Keeler and Backofen [12] and Goodwin [13]. The mechanical behavior of both layers is assumed to be isotropic, incompressible and strain-rate independent. The elastomer layer is modeled by a neo-Hookean constitutive law, while the metal layer is assumed to follow the rigidplastic flow theory. Indeed, because necking occurs at a relatively high plastic strain level, the elasticity of the metal layer may be neglected. This choice allows significantly simplifying the modeling and the numerical resolution of the problem. A mixed (isotropic and kinematic) hardening model is used to describe the evolution of the yield surface of the metal layer. The isotropic hardening contribution is modeled by the Hollomon law, while the Armstrong-Frederick model is used to describe kinematic hardening. It must be noted that the main novelty of the current work, compared to a previous contribution by the same authors [7], is the introduction of kinematic hardening into the constitutive modeling of the metal layer. The consideration of kinematic hardening is essential to the accurate constitutive modeling of a 
number of metallic materials, such as dual phase (DP) steels. Indeed, without inclusion of kinematic hardening within the constitutive framework, it would not be possible to account for some important physical phenomena, such as the Bauschinger effect. The latter may result from high internal stresses induced by the heterogeneity contrast between different metallurgical phases (e.g., hard martensitic islands embedded in a ductile ferritic matrix).

To predict the ductility limit of metallic materials, various strain localization criteria have been proposed in the literature. In this regard, we can quote the bifurcation analysis [14-16] and the imperfection approach $[17,18]$. Within the current constitutive framework for the metal layer (flow theory of plasticity), which does not involve essential destabilizing mechanisms such as yield-surface vertex effects, the bifurcation theory would predict unrealistically high limit strains in the right-hand side of the FLD (see, e.g. $[7,18,19]$ ). In contrast to the bifurcation analysis, the imperfection approach allows predicting ductility limits at realistic strain levels, due to the assumed geometric or material imperfection that plays a major destabilizing role, which systematically precipitates early localization of plastic strain. For these reasons, the latter approach, which has been often shown to be an interesting alternative to the bifurcation theory (see, e.g. [7]), is followed in the present paper. It postulates the preexistence of a small initial imperfection in the form of a narrow band across the studied metal layer. This imperfection may be assumed as local variations in thickness (geometric imperfection) or in plastic properties (material imperfection), which will affect the plastic flow and therefore influence the occurrence of strain localization. This approach, which was initially introduced by Marciniak and Kuczynski [17], will be referred to hereafter as the $\mathrm{M}-\mathrm{K}$ analysis. Note that in its original version, it was developed within a simple constitutive framework, namely an isotropic rigid-plastic model with isotropic hardening (i.e., Hollomon law). In spite of the over-sensitivity of its predictions to the initial imperfection value (see, e.g. [20]), the M-K analysis has attracted a great deal of attention, due to its pragmatic character and its versatility. Indeed, to improve the accuracy of the FLD predictions, this approach has been combined with various behavior models, including strain-rate sensitivity [18,21], plastic anisotropy [22-24], and damage-induced softening [25-27]. It was also extended to kinematic hardening (see [19]) in order to predict localized necking in biaxially stretched sheets. However, in that earlier study, the biaxial loading ratio was restricted to the range of positive biaxial stretching. Despite this limitation, it was shown in that former investigation that the FLDs predicted by kinematic hardening are in far better agreement with experimental results than their counterparts yielded by standard flow theory with isotropic hardening. Subsequently, the imperfection approach has been coupled by Hutchinson and Tvergaard [28] with a constitutive model that accounts for kinematic hardening for a detailed analysis of shear band formation, although restricted to plane-strain tension. Note that the impact of kinematic hardening on the shape and level of FLDs predicted by $\mathrm{M}-\mathrm{K}$ analysis has also been investigated in the literature. In this regard, we can quote the contributions of Chu [29], Lu and Lee [30] and Butuc et al. [31]. Except for very few papers, it is generally shown that kinematic hardening leads to a decrease in the limit strains, especially for strain paths in the neighborhood of equibiaxial tension $[19,30,32]$. In the present paper, the effect of kinematic hardening on the ductility of both freestanding and substrate-supported metal layers is investigated. As will be revealed by various numerical simulations, kinematic hardening is shown to generally decrease the limit strains. This result, which is obviously dependent on the parameters associated with the kinematic hardening model, is in reasonable agreement with previously reported works. To investigate the influence of an elastomer substrate, the $\mathrm{M}-\mathrm{K}$ analysis is extended to the prediction of FLDs for substrate-supported metal layers. The two layers (metal and elastomer) are assumed to be perfectly adhered (i.e., no delamination is allowed). Consistent with the results of a previous study [7], which was carried out for metal layers with only isotropic hardening, it is found that the presence of an elastomer substrate substantially enhances the necking limit of the resulting metal/elastomer bilayer.

The reminder of the paper is organized as follows:

- Section 2 outlines the constitutive equations that model the behavior of the metal and elastomer layers.

- Section 3 details the imperfection approach adopted to predict localized necking in the bilayer.

- Section 4 deals with the algorithm used to predict the forming limit diagrams.

- The various numerical predictions are presented in Section 5, where the effects on localized necking of kinematic hardening and of the addition of an elastomer layer are discussed in details. Comparisons with experimental results are also carried out to assess the relevance of incorporating kinematic hardening in the constitutive modeling of freestanding metal sheets.

\section{Notations, conventions and abbreviations}

The derivations presented in this paper are carried out using classic conventions. Note that the assorted notations can be combined, while additional notations will be clarified as needed following related equations.

Vectorial and tensorial fields are designated by bold letters and symbols.

Scalar variables and parameters are represented by thin italic letters and symbols.

Einstein's convention of summation over repeated indices is adopted. The range of the free (resp. dummy) index is given before (resp. after) the corresponding equation.

- time derivative of $\bullet$

${ }^{T}$ transpose of tensor $\bullet$

$\vec{\bullet} \otimes \vec{\bullet}$ tensor product of two vectors $\left(=\bullet_{i} \bullet_{j}\right)$

- value of quantity $\bullet$ at the initial time.

$\operatorname{Tr}(\bullet)$ trace of second-order tensor $\bullet$

- value of quantity $\bullet$ at time $t$ (for convenience, the dependence on time is most often omitted when the variable is expressed in the current instant).

$\bullet(*)$ quantity $\bullet$ associated with behavior in layer *

${ }^{B}$ quantity $\bullet$ associated with behavior in the band.

- $S$ quantity $\bullet$ associated with behavior in the safe zone.

\section{Constitutive equations}

\subsection{Metal layer}

The mechanical behavior of the metal layer is assumed to follow the flow theory of plasticity. Because strain localization occurs at relatively large strains, elasticity may be neglected and, hence, the behavior is taken rigid-plastic. The flow rule is defined by the normality relationship

$\dot{\varepsilon}=\dot{\lambda} \frac{\partial F_{p}}{\partial \sigma}$

where $\dot{\lambda}$ is the plastic multiplier (equal to the equivalent strain rate $\dot{\varepsilon}_{e q}$ ) and $F_{p}$ is the yield function.

Plasticity is assumed to be isotropic following the von Mises criterion. Furthermore, the evolution of the radius of the yield surface is described by an isotropic hardening law, while its translation is governed by a kinematic hardening law. This leads to the following expression for the yield function $F_{p}$ :

$F_{p}=\sqrt{(3 / 2)\left(\boldsymbol{\sigma}_{d}-\mathbf{X}\right):\left(\boldsymbol{\sigma}_{d}-\mathbf{X}\right)}-\sigma_{Y}=0$,

where

$\boldsymbol{\sigma}_{d}$ denotes the deviatoric part of the Cauchy stress tensor $\boldsymbol{\sigma}$, $\mathbf{X}$ is the back-stress tensor, which describes the yield surface translation, 
$\sigma_{Y}$ is the yield stress, which measures the evolution of the size of the yield surface.

With the Hollomon isotropic hardening model, the yield stress $\sigma_{Y}$ is related to the equivalent strain $\varepsilon_{e q}$ by the following power law:

$\sigma_{Y}=K \varepsilon_{e q}^{n}$.

The evolution law of the back-stress $\mathbf{X}$ is defined by the ArmstrongFrederick kinematic hardening model [33]

$\dot{\mathbf{X}}=C_{x}\left(X_{s a t} \dot{\varepsilon}-\dot{\varepsilon}_{e q} \mathbf{X}\right)$.

In Eqs. (3) and (4) above, $K, n, C_{x}$, and $X_{s a t}$ are material parameters. By substituting Eq. (2) into Eq. (1), the normality rule can be rewritten as

$\dot{\varepsilon}=\dot{\varepsilon}_{e q} \frac{3\left(\boldsymbol{\sigma}_{d}-\mathbf{X}\right)}{2 \sigma_{Y}}$.

From Eq. (5), it is easy to determine the expression of $\sigma_{d}$

$\boldsymbol{\sigma}_{d}=\mathbf{X}+\frac{2 \sigma_{Y}}{3 \dot{\varepsilon}_{e q}} \dot{\boldsymbol{\varepsilon}}$

\subsection{Elastomer layer}

The neo-Hookean model adopted in this paper to describe the mechanical behavior of the elastomer substrate is defined by the following constitutive equation $[34,35]$ :

$\mathbf{B}=\mu \mathbf{V}^{2}$

where $\mathbf{B}$ is a stress tensor (related to the Cauchy stress tensor $\boldsymbol{\sigma}$ by Eq. (9)), $\mu$ is the shear modulus and $\mathbf{V}$ is the left Cauchy-Green tensor defined by the following relation:

$\mathbf{V}^{2}=\mathbf{F} \mathbf{F}^{T}$

with $\mathbf{F}$ being the deformation gradient tensor.

The constitutive law (7) is selected among other models [36-38], because it furnishes a valid description for the finite elastic behavior of many real rubber-like materials, provided that the deformations are not too extreme.

\subsection{Plane-stress condition}

The Cauchy stress tensor $\boldsymbol{\sigma}$ corresponding to the metal (resp. elastomer) layer is expressed as a function of $\boldsymbol{\sigma}_{d}$ (resp. B) given in Eq. (6) (resp. (7)) and the hydrostatic pressure $p=(1 / 3) \operatorname{Tr}(\boldsymbol{\sigma})$ (resp. an unknown pressure $q$ ) as follows:

$$
\text { metal layer: } \quad \boldsymbol{\sigma}=\boldsymbol{\sigma}_{d}+p \text { Id }
$$$$
\text { elastomer layer: } \quad \boldsymbol{\sigma}=\mathbf{B}+q \mathbf{I d} \text {, }
$$

where Id is the second-order identity tensor.

Consistent with several literature works and because the bilayer is assumed to be thin, the assumption of generalized plane stress will be adopted in the M-K analyses. Hutchinson et al. [39] have proven the validity of such an assumption in the case of thin media. Under this condition, the stress components normal to the bilayer $\sigma_{i 3} ; i=1,2,3$ are always zero. Thus, pressures $p$ and $q$ can be eliminated from Eq. (9) by means of relation $\sigma_{33}=0$, which gives after straightforward calculation

metal layer:

$$
p=\sigma_{d 11}+\sigma_{d 22}
$$

elastomer layer: $q=-B_{33}$.

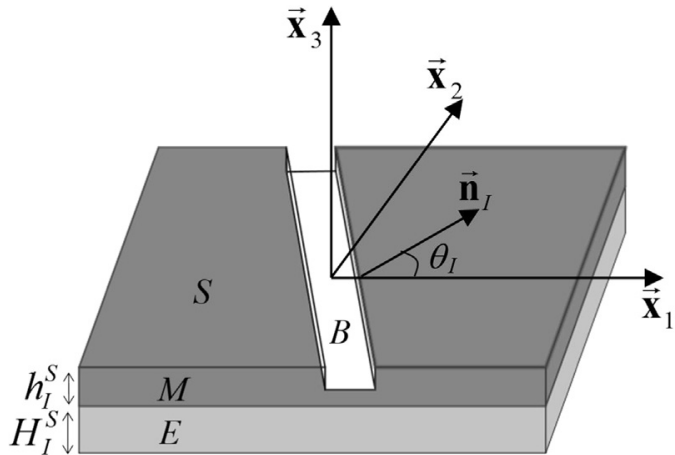

Fig. 1. Illustration of the $\mathrm{M}-\mathrm{K}$ analysis for a bilayer (initial geometry and band orientation).

\section{Imperfection approach}

\subsection{Governing equations for the imperfection approach}

The prediction of localized necking in substrate-supported metal layers is carried out using the M-K approach. The initial imperfection (in the form of a groove), required for the $\mathrm{M}-\mathrm{K}$ analysis, is assumed to initiate within the metal layer.

Fig. 1 depicts the bilayer in its initial reference configuration (i.e., the state before application of loading). In the sequel, the following notations will be employed:

- $h_{I}^{B}$ : initial thickness of the metal layer M inside the band $\mathrm{B}$.

- $h_{I}^{S}$ : initial thickness of the metal layer $\mathrm{M}$ in the safe zone $\mathrm{S}$ (outside the band).

- $H_{I}^{B}$ : initial thickness of the elastomer layer $\mathrm{E}$ inside the band B.

- $H_{I}^{S}$ : initial thickness of the elastomer layer $\mathrm{E}$ in the safe zone $\mathrm{S}$ (outside the band).

- $\overrightarrow{\mathbf{n}}_{I}$ : initial unit normal to the band.

- $\theta_{I}$ : initial orientation of the band.

According to the notations above, the initial imperfection may be measured by a factor $\xi_{I}$, defined as

$\xi_{I}=1-\frac{h_{I}^{B}}{h_{I}^{S}}$,

which will be used in what follows.

Another assumption, adopted in the subsequent $\mathrm{M}-\mathrm{K}$ analysis, is that the two layers remain perfectly adhered (i.e., delamination is not considered in the current study). This condition can be expressed by the equality of the velocity gradient tensors (denoted $\mathbf{G}$ in what follows), during loading, between the metal and the elastomer layers (both in the band and in the safe zone)

$\mathbf{G}^{B}(M)=\mathbf{G}^{B}(E)=\mathbf{G}^{B} \quad ; \quad \mathbf{G}^{S}(M)=\mathbf{G}^{S}(E)=\mathbf{G}^{S}$.

In addition to condition (12), the $\mathrm{M}-\mathrm{K}$ analysis is defined by the following relations and considerations:

- As illustrated in Fig. 1, strain localization is interpreted here as a jump in the velocity gradient over a planar band in the plane of the sheet. The initial imperfection approach is based on the condition that kinematic compatibility between the band and the safe zone must be satisfied. This compatibility condition requires the jump in the velocity gradient to be in the form

$\left\|\frac{\partial \mathbf{v}}{\partial \mathbf{x}}\right\|=\overrightarrow{\mathbf{c}} \otimes \overrightarrow{\mathbf{n}}$ with $\llbracket \frac{\partial \mathbf{v}}{\partial \mathbf{x}} \|=\frac{\partial \mathbf{v}^{B}}{\partial \mathbf{x}}-\frac{\partial \mathbf{v}^{S}}{\partial \mathbf{x}}$,

where $\mathbf{x}$ is the current position of a material point, $\overrightarrow{\mathbf{c}}$ denotes a jump vector, and $\overrightarrow{\mathbf{n}}$ designates the current unit vector normal to the band 
(its counterpart $\overrightarrow{\mathbf{n}}_{I}$ in the initial configuration is shown in Fig. 1). By adopting again the above tensor notation $\mathbf{G}$ for the velocity gradient, Eq. (13) can be equivalently rewritten as follows:

$\mathbf{G}^{B}=\mathbf{G}^{S}+\overrightarrow{\dot{\mathbf{c}}} \otimes \overrightarrow{\mathbf{n}}$.

- The equilibrium of the normal and shear forces across the imperfection band is also maintained throughout the deformation. This equilibrium can be expressed in terms of Cauchy stress tensors by the following relation:

$\left(h^{B} \boldsymbol{\sigma}^{B}(M)+H^{B} \boldsymbol{\sigma}^{B}(E)\right) \cdot \overrightarrow{\mathbf{n}}=\left(h^{S} \boldsymbol{\sigma}^{S}(M)+H^{S} \boldsymbol{\sigma}^{S}(E)\right) \cdot \overrightarrow{\mathbf{n}}$.

- The evolution of the band orientation, which is given by Nanson's relation

$\operatorname{Tan}(\theta)=e^{\left(\varepsilon_{11}^{S}-\varepsilon_{22}^{S}\right)} \operatorname{Tan}\left(\theta_{I}\right)$.

- The evolution of the current measure of imperfection, which is given by factor $\xi$

$\xi=1-\frac{h^{B}}{h^{S}}$.

Making use of the following relations

$h^{B}=h_{I}^{B} e^{\varepsilon_{33}^{B}} \quad ; \quad h^{S}=h_{I}^{S} e^{\varepsilon_{33}^{S}}$,

factor $\xi$ can then be expressed as

$\xi=1-\left(1-\xi_{I}\right) e^{\left(\varepsilon_{33}^{B}-\varepsilon_{33}^{S}\right)}$.

- The constitutive Eqs. (1)-(5) for the metal layer, and (7)-(8) for the elastomer layer.

\subsection{Velocity gradient tensors in the band and in the safe zone}

For the FLD prediction, proportional strain paths are prescribed to the safe zone of the bilayer as follows:

$\frac{G_{22}^{S}}{G_{11}^{S}}=\frac{\dot{\varepsilon}_{22}^{S}}{\dot{\varepsilon}_{11}^{S}}=\rho=$ constant.

The strain ratio $\rho$ is varied in the range $-1 / 2 \leq \rho \leq 1$ to span the complete FLD.

Exploiting the incompressibility condition of the metal and elastomer layers along with the plane-stress conditions, and making use of Eq. (20), the velocity gradient in the safe zone can be expressed as a function of $\rho$ and $\dot{\varepsilon}_{11}^{S}$

$\mathbf{G}^{S}=\left(\begin{array}{ccc}\dot{\varepsilon}_{11}^{S} & 0 & 0 \\ 0 & \rho \dot{\varepsilon}_{11}^{S} & 0 \\ 0 & 0 & -(1+\rho) \dot{\varepsilon}_{11}^{S}\end{array}\right)$.

Then, from Eq. (14), the components of the velocity gradient in the band can be expressed in the following form:

$G_{11}^{B}=\dot{\varepsilon}_{11}^{S}+\dot{c}_{1} n_{1} ; G_{22}^{B}=\rho \dot{\varepsilon}_{11}^{S}+\dot{c}_{2} n_{2} ;$

$G_{33}^{B}=-(1+\rho) \dot{\varepsilon}_{11}^{S}-\dot{c}_{1} n_{1}-\dot{c}_{2} n_{2} ;$

$G_{12}^{B}=\dot{c}_{1} n_{2} ; G_{21}^{B}=\dot{c}_{2} n_{1}$;

$G_{13}^{B}=G_{23}^{B}=G_{31}^{B}=G_{32}^{B}=0$.

$\overrightarrow{\mathbf{n}}$.

\section{Algorithm for the FLD prediction}

The general algorithm used to predict the FLD of the elastomer/ metal bilayer is based on the following three nested loops:

- For $\rho=-1 / 2$ to $\rho=1$ at user-defined intervals (here, we take intervals of 0.1 ).

- For $\theta_{I}$ spanning the admissible range of inclination angle (i.e., between $0^{\circ}$ and $90^{\circ}$ ), at user-defined intervals (here, we take intervals of $1^{\circ}$ ).

- For each time increment $\left[\begin{array}{ll}t_{n}, & t_{n+1}\end{array}\right]$ (with $t_{n+1}=t_{n}+\Delta t$ ), apply the implicit incremental algorithm described in Appendix A to integrate the governing equations of the metal and elastomer layers both in the safe zone and in the band. The application of this incremental integration scheme is stopped when the following criterion is reached:

$G_{33}^{B} / G_{33}^{S} \geq 10$.

The strain component $\varepsilon_{11}^{S}$, thus obtained once the criterion (23) is satisfied, is considered to be the critical strain $\varepsilon_{11}^{*}$ corresponding to the current band inclination $\theta$ and strain path $\rho$.

The smallest critical strain $\varepsilon_{11}^{*}$, solution of the above algorithm, over all initial angles $\theta_{I}$ and the corresponding current angle define, respectively, the necking limit strain $\varepsilon_{11}^{L}$ and the necking band orientation for the current strain ratio $\rho$.

\section{Results and discussions}

This section is divided into three main sections:

- Section 5.1, which corresponds to the numerical results relating to the freestanding metal layer.

- Section 5.2, which provides comparisons between the current numerical predictions and some experimental results taken from the literature. The main objective of these comparisons is to analyze the relevance of incorporating kinematic hardening in the constitutive modeling of freestanding metal sheets.

- Section 5.3, which contains the numerical results pertaining to the elastomer/metal bilayer.

\subsection{Freestanding metal layer}

The material parameters relating to the metal layer that are used in Sections 5.1 and 5.3 are those of a DC06 steel [31]. These parameters have been identified according to the procedure detailed in 3DS Report [40]. The values corresponding to these material parameters are reported in Table 1.

Note that the parameters of Table 1 have been fitted in order to obtain the same stress-strain response for both isotropic and mixed hardening models, as shown in Fig. 2.

In order to emphasize the effect of the hardening modeling on localized necking, we plot in Fig. 3 the evolution of the ratio $\varepsilon_{11}^{B} / \varepsilon_{11}^{S}$ as a function of $\varepsilon_{11}^{S}$, for three representative in-plane loading paths: uniaxial tension $(\rho=-0.5)$, plane-strain tension $(\rho=0)$, and equibiaxial tension $(\rho=1)$. This evolution can be used as a reliable indicator for the onset of localized necking. The different curves are stopped when the necking criterion (23) is satisfied. From these curves, the following

Table 1

Material parameters for the DC06 steel.

\begin{tabular}{lllll}
\hline & $K(\mathrm{MPa})$ & $n$ & $C_{x}$ & $X_{\text {sat }}(\mathrm{MPa})$ \\
\hline Isotropic hardening & 522 & 0.257 & & \\
Mixed hardening & 447 & 0.221 & 1.87 & 58.1 \\
\hline
\end{tabular}




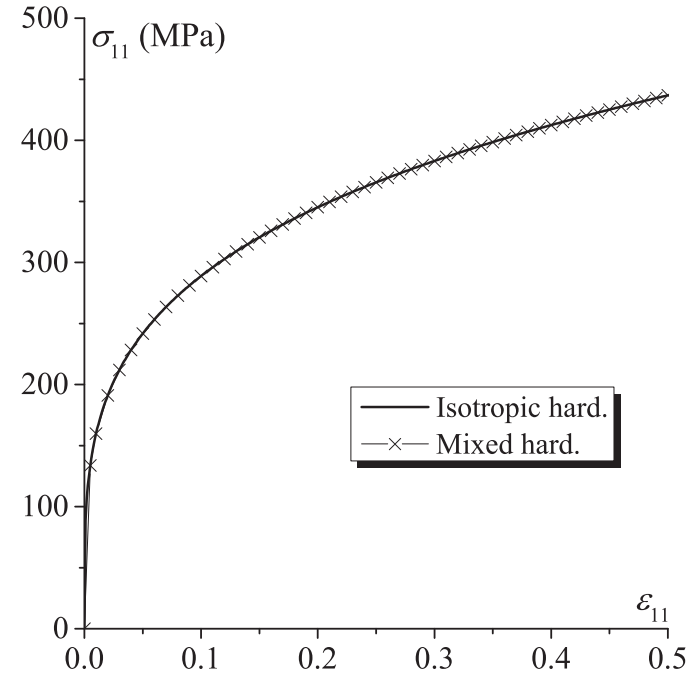

Fig. 2. Stress-strain response corresponding to a uniaxial tensile test: comparison between the isotropic and mixed hardening models.

conclusions can be drawn:

- Although the uniaxial stress-strain curves are the same for both hardening models (isotropic and mixed), as displayed in Fig. 2, the evolution of the ratio $\varepsilon_{11}^{B} / \varepsilon_{11}^{S}$ as a function of $\varepsilon_{11}^{S}$ differs from one hardening model to another, as shown in Fig. 3. This difference is due to the fact that the strain path inside the band is nonproportional during the deformation. Indeed, at the beginning of the loading, the scalar components of the jump vector $\overrightarrow{\overrightarrow{\mathbf{c}}}$ are very close to zero. Hence, the strain field (and consequently the strain path) inside the band is very close to its counterpart outside the band (see Eq. (14)). As deformation proceeds, the strain path inside the band gradually deviates from proportionality and ultimately switches to a plane-strain tensile state at the onset of localized necking (regardless the value of $\rho$ ). Therefore, although both hardening models provide the same uniaxial stress-strain response, they yield different mechanical responses inside the band (due to the complexity in the loading path). These differences clearly explain why the predicted limit strains are influenced by the hardening model considered.

- In the particular case of plane-strain tension $(\rho=0)$, both hardening models should predict the same limit strain, as the strain path remains proportional both in the safe zone and in the band. The slight differences between the limit strains for plane-strain tension $(\rho=0)$ predicted by the isotropic and the mixed hardening models, as observed in Fig. 3(c) and Fig. 3(d), are due to the inaccuracy in the identification of the hardening parameters for the isotropic model.

- The critical strain $\varepsilon_{11}^{*}$ can be defined as the maximum value of $\varepsilon_{11}^{S}$ reached during loading. The kinematic hardening has an influence on the evolution of the strain component $\varepsilon_{11}^{S}$, and thus on the critical strain $\varepsilon_{11}^{*}$, as demonstrated in Fig. 3. Indeed, for the uniaxial tensile state and the plane-strain state, the kinematic hardening leads to a relative increase in $\varepsilon_{11}^{*}$, as shown in Fig. 3(a), (b), (c) and (d). For the equibiaxial loading path $(\rho=1)$, the kinematic hardening decreases the predicted value of the critical strain $\varepsilon_{11}^{*}$, as shown in Fig. 3(e), (f).

- The value of the initial imperfection factor $\xi_{I}$ does not affect the dependence of the critical strain $\varepsilon_{11}^{*}$ on kinematic hardening. However, it affects the level of the predicted critical strain $\varepsilon_{11}^{*}$. Indeed, the value of the critical strain $\varepsilon_{11}^{*}$ decreases when the imperfection factor $\xi_{I}$ increases. This result is consistent with previously reported works using the $\mathrm{M}-\mathrm{K}$ analysis.

- At the beginning of loading, the imperfection factor is very small (about $5 \times 10^{-3}-10^{-2}$ ), and hence the thicknesses of the band and of the safe zone are quite comparable. For this reason, the ratio $\varepsilon_{11}^{B} / \varepsilon_{11}^{S}$ is very close to 1 . As deformation increases, the imperfection starts to grow in magnitude, resulting in a decreasing thickness in the band, a phenomenon that becomes very rapid as soon as localization initiates. This phenomenon is also clearly reflected by the abrupt evolution of the ratio $\varepsilon_{11}^{B} / \varepsilon_{11}^{S}$, as demonstrated in the different curves of Fig. 3. This fast increase in the ratio $\varepsilon_{11}^{B} / \varepsilon_{11}^{S}$, at the approach of localization, implies that the predicted critical strain $\varepsilon_{11}^{*}$ is not sensitive to the threshold criterion used in Eq. (23). Therefore, changing the value of this threshold from 10 to 50, for example, does not affect the level of $\varepsilon_{11}^{*}$.

Fig. 4 plots the evolution of the critical strain $\varepsilon_{11}^{*}$ as a function of the initial band orientation $\theta_{I}$ for three representative strain paths: $\rho=-0.5, \rho=0$ and $\rho=1$. Localized necking occurs along the most critical orientation that corresponds to the lowest critical strain level. The conclusions suggested by Fig. 4 are given in what follows for the different strain paths investigated:

- Uniaxial tension ( $\rho=-0.5$ ): For both hardening models (isotropic and mixed), the critical strain decreases as a function of $\theta_{I}$, until reaching its lowest value, and increases afterwards. This result is consistent with several existing literature studies (see, e.g. [6,7]).

- Plane-strain tension $(\rho=0)$ : The lowest critical strain for the occurrence of necking always corresponds to $\theta_{I}=0$, as shown in Fig. 4(c), (d). In other words, strain localization always initiates and develops along a band perpendicular to the plane-strain tension direction. This latter conclusion applies to all of the strain paths ranging from plane-strain tension $(\rho=0)$ to equibiaxial tension $(\rho=1)$, and is valid for both hardening models.

- Equibiaxial tension $(\rho=1)$ : The value of the critical strain is independent of the initial inclination angle. However, it obviously depends on the material parameters and on the initial imperfection factor. In other words, under balanced biaxial tension, necking could occur along any orientation, once the applied loading reaches the necking criterion (23). This observation is attributable to the symmetry of the problem under equibiaxial tension (i.e., no preferential direction, whenever isotropic plasticity is assumed), and confirms previous conclusions drawn by Jia and Li [6]. Note that in this latter reference, the bifurcation approach was used as necking criterion, instead of the $\mathrm{M}-\mathrm{K}$ imperfection analysis; and the $\mathrm{J}_{2}$ deformation theory of plasticity was adopted as constitutive modeling, rather than the flow theory of plasticity.

The effect of the initial imperfection factor $\xi_{I}$ on the shape and the level of the FLDs is investigated in Fig. 5 for both hardening models. As well-known from previously reported works based on the M-K analysis, the effect of the initial imperfection is essentially to shift the FLD downwards. Accordingly, the level of FLD is lowered when the value of $\xi_{I}$ increases. For both hardening models, the imperfection plays a destabilizing role that precipitates the occurrence of strain localization. It is noteworthy that the introduction of kinematic hardening in the constitutive modeling does not modify this general trend. It is also observed from Fig. 5 that the limit strains are more sensitive to the initial imperfection in the range of positive biaxial stretching than in the range of negative minor strains. Analyzing the limit strain level for strain-path ratios close to 1 (i.e., in the neighborhood of equibiaxial tension), it is clear that localization cannot be predicted at realistic strain levels for this range of strain paths without introducing initial imperfections.

The effect of kinematic hardening on the FLDs and on the necking band orientations is studied in Fig. 6. The three values used in Fig. 5 for the initial imperfection factor are considered here once again; namely, $10^{-3}, 5 \times 10^{-3}$ and $10^{-2}$. The dependence of FLDs on kinematic hardening revealed by Fig. 6 is consistent with the results of Figs. 3, 4 and 5. When kinematic hardening is considered, the limit strains increase slightly in 


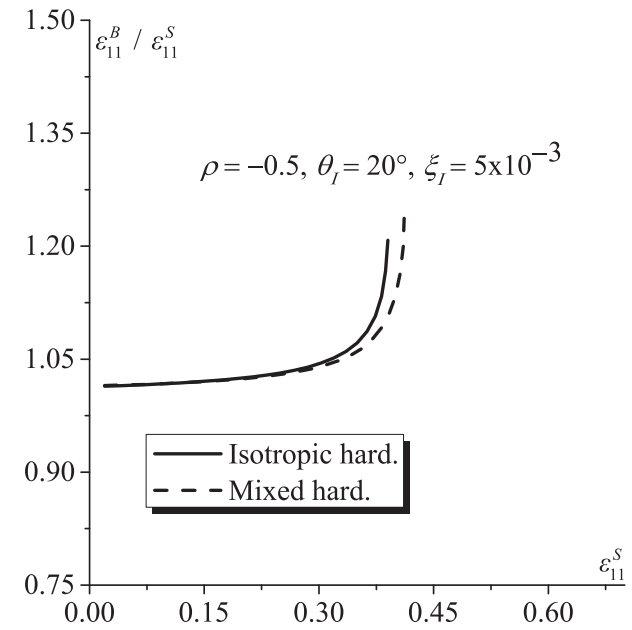

(a)

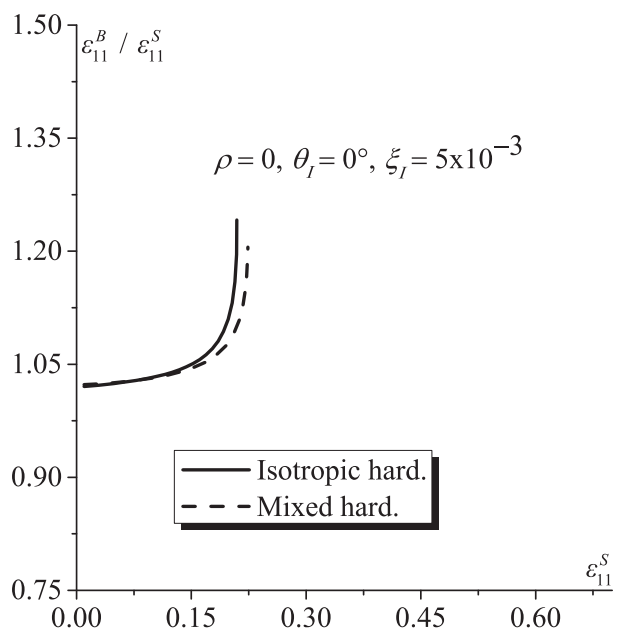

(c)

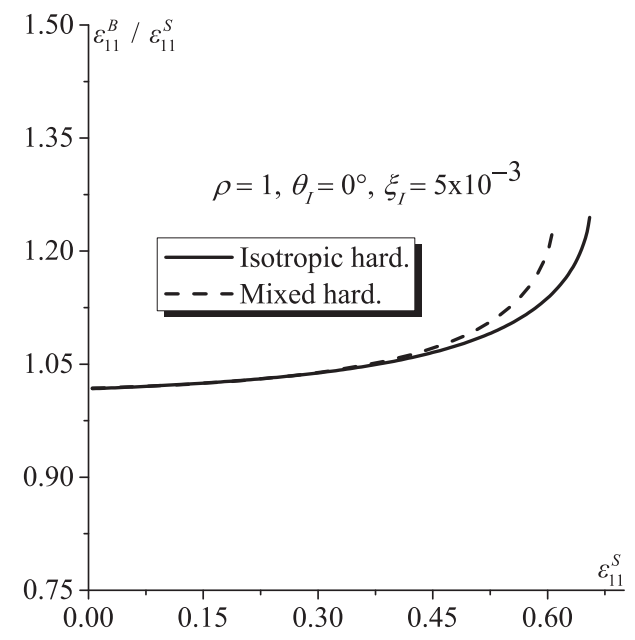

(e)

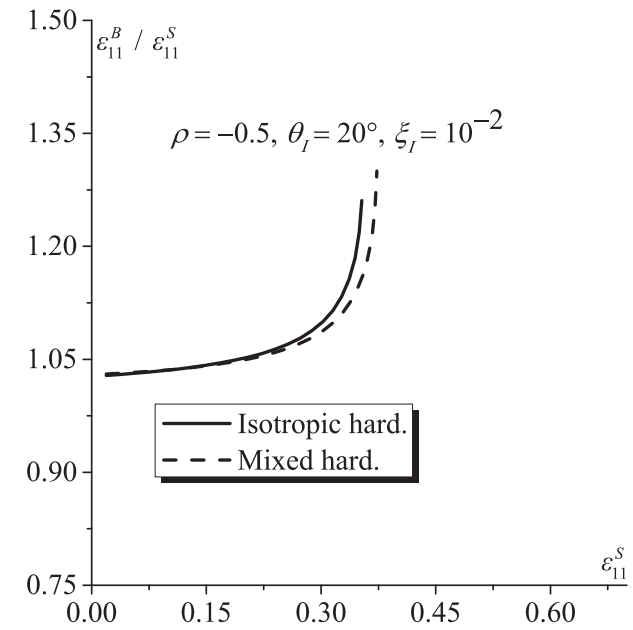

(b)

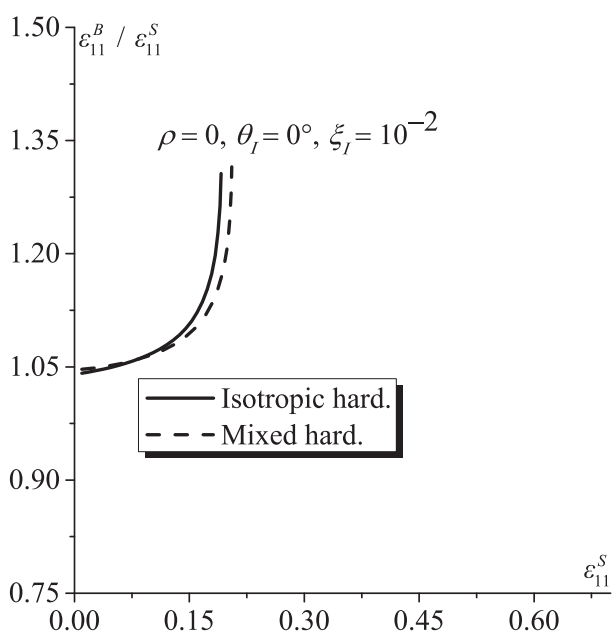

(d)

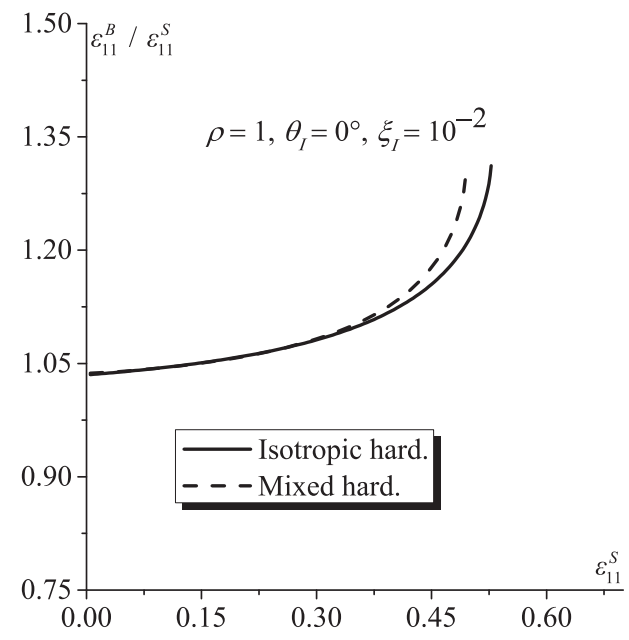

(f)

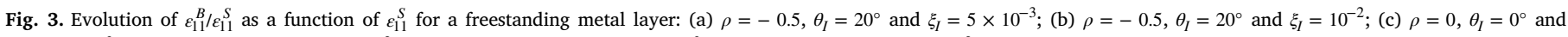
$\xi_{I}=5 \times 10^{-3}$; (d) $\rho=0, \theta_{I}=0^{\circ}$ and $\xi_{I}=10^{-2}$; (e) $\rho=1, \theta_{I}=0^{\circ}$ and $\xi_{I}=5 \times 10^{-3}$; (f) $\rho=1, \theta_{I}=0^{\circ}$ and $\xi_{I}=10^{-2}$.

the range of negative minor strains, while they decrease more visibly in the range of positive biaxial stretching. This observation is valid for the different initial imperfection factors considered. To sum up, it can be concluded that kinematic hardening has an effective impact on the predicted forming limits; however, the observed influence is relatively small in the whole. This relatively small overall effect may be attributed 


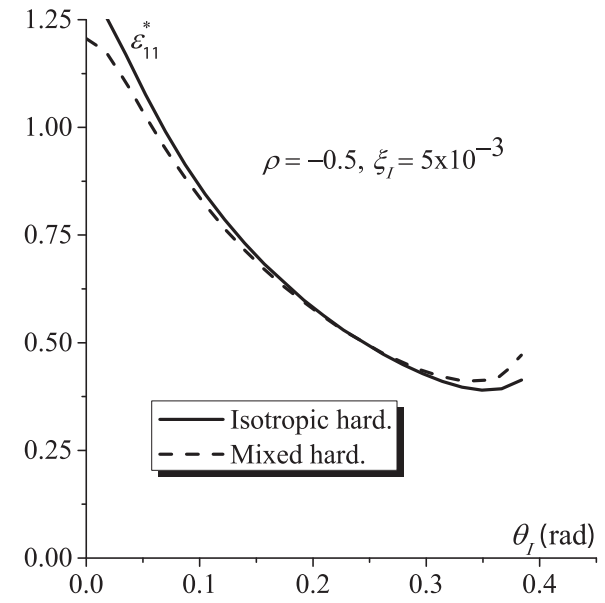

(a)

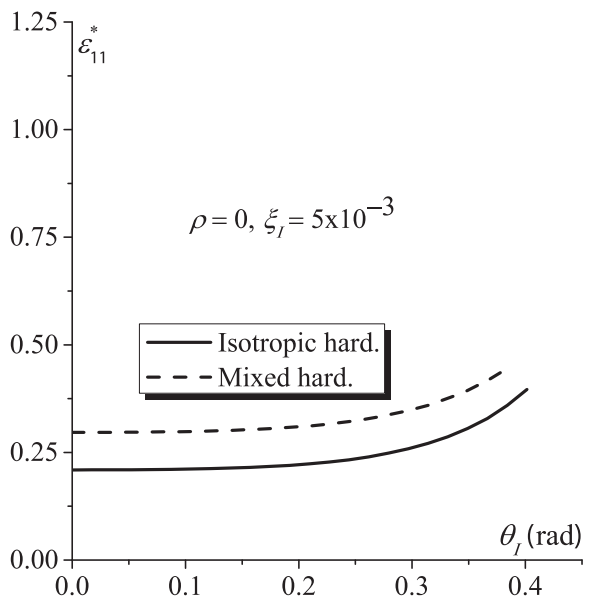

(c)

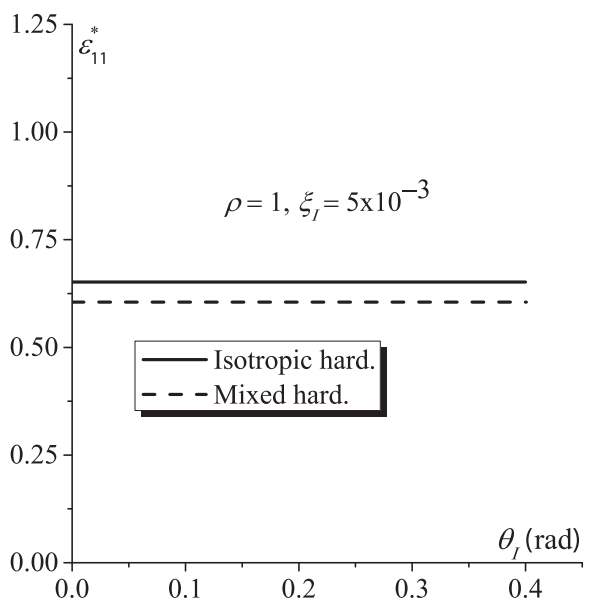

(e)

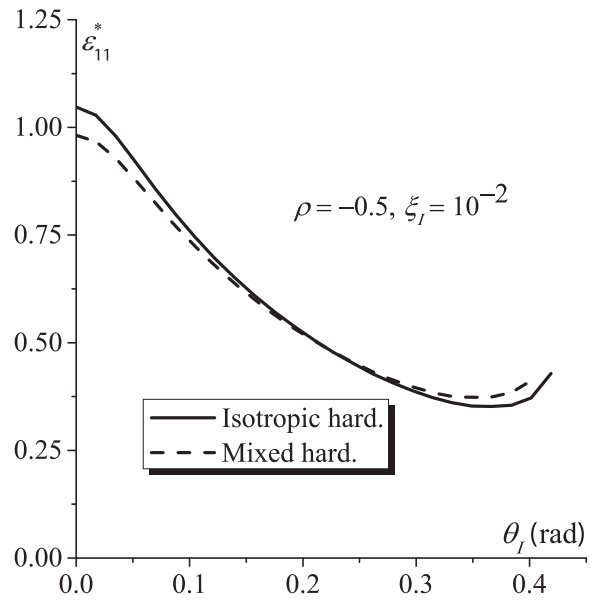

(b)

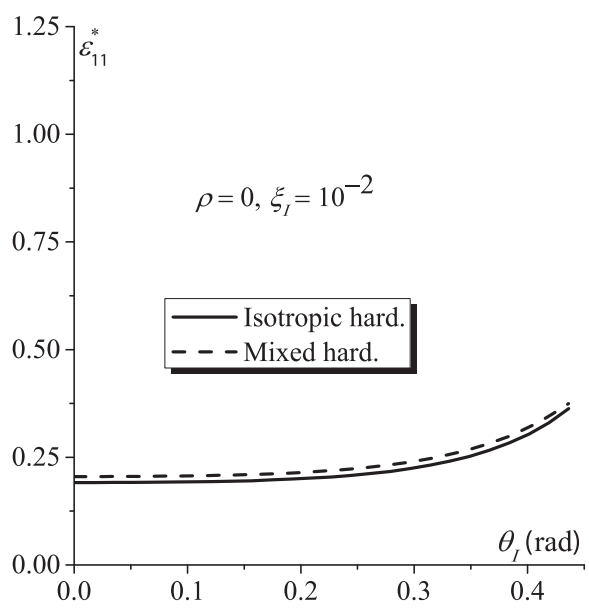

(d)

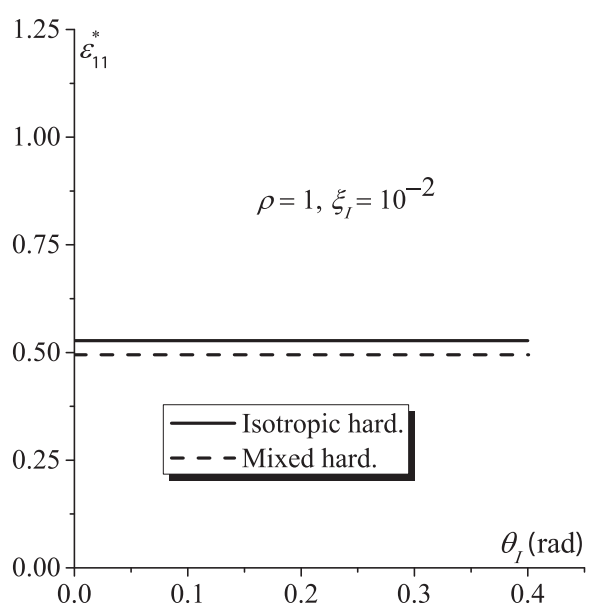

(f)

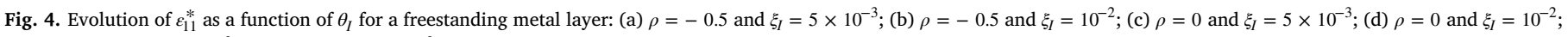
(e) $\rho=1$ and $\xi_{I}=5 \times 10^{-3}$; (f) $\rho=1$ and $\xi_{I}=10^{-2}$.

to the small difference in the yield surfaces corresponding to the two hardening models.

In terms of band orientation, the different simulations show that for positive strain paths $(\rho \geq 0)$, the necking band is always perpendicular to the major strain direction. Consequently, this range of strain paths is not considered in Fig. 6 . In the range of negative strain paths $(\rho<0)$, however, the necking band is generally predicted with a slanted direction, lying between the two loading directions, as demonstrated in Fig. 6. The corresponding inclination angle is shown to increase as the strain-path ratio $\rho$ decreases (see Fig. 6(b), (d) and (f)). It is also clear from these figures that the inclination angle is dependent on the hardening model; the inclination angle predicted by the mixed hard- 


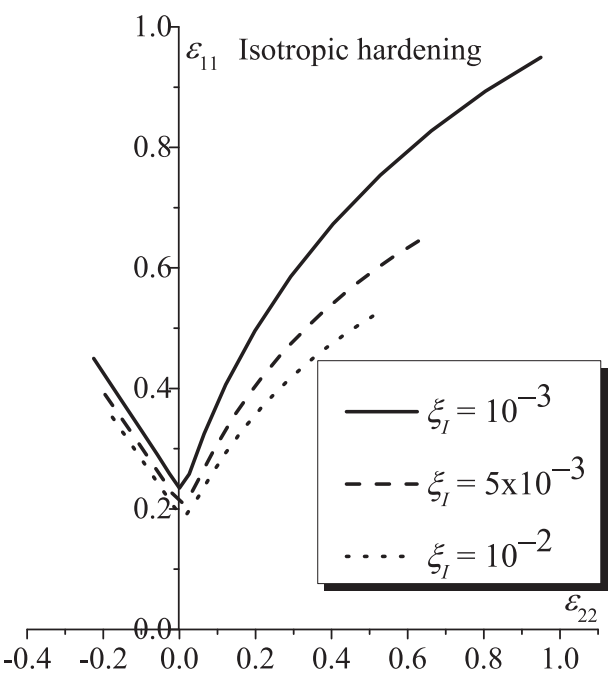

(a)

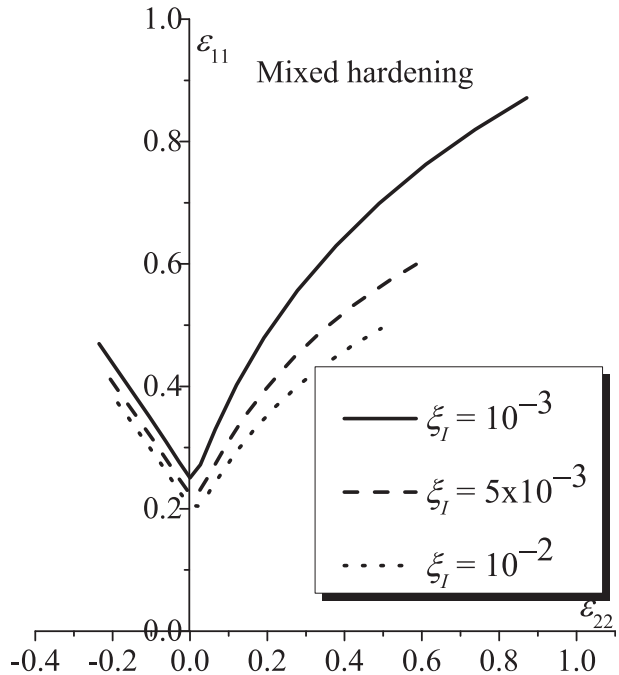

(b)

Fig. 5. Effect of the initial imperfection factor on the FLDs for a freestanding metal layer: (a) Isotropic hardening; (b) Mixed hardening.

ening model is found generally lower than that predicted by its isotropic hardening counterpart.

It is worth noting that for all of the previous results, the hardening parameters were calibrated on the basis of experimental tests. Accordingly, the identified value for the back-stress saturation $X_{\text {sat }}$ was set to $58.1 \mathrm{MPa}$ (see Table 1). This value is rather small, because for an equivalent strain $\varepsilon_{e q}=0.5$, the value of the back-stress saturation $X_{\text {sat }}$ is only about $13 \%$ of the yield stress $\sigma_{Y}$. Therefore, the translation of the yield surface is relatively small compared to its expansion. This explains the results revealed by Fig. 6, where the effect of kinematic hardening on the limit strains was not found sufficiently significant. In order to further investigate the effect of kinematic hardening on the FLDs, a parametric study is carried out. In this parametric study, four fictitious materials are considered. For each fictitious material, two sets of parameters are considered: the first set corresponds to the isotropic hardening model, while the second is associated with the mixed hardening model. The parameters $K, n$ and $C_{x}$, corresponding to the mixed hardening model, are kept identical for all materials (as detailed in Table 2), and only the saturation parameter $X_{\text {sat }}$ is varied from one material to another (100 MPa, $200 \mathrm{MPa}, 300 \mathrm{MPa}$ and $400 \mathrm{MPa}$ ). Once the parameters corresponding to the mixed hardening model are fixed, the hardening parameters $K$ and $n$ for the isotropic hardening model are fitted in order to obtain the same uniaxial stress-strain response yielded by the two hardening models for each fictitious material. The material parameters, thus identified for all materials and both hardening models, are given in Table 2 .

The effect of the kinematic hardening saturation parameter $X_{\text {sat }}$ on the shape and the level of the FLDs is illustrated in Fig. 7. For the different simulations reported in Fig. 7, the initial imperfection factor $\xi_{I}$ is fixed to $10^{-2}$. It is clearly shown that the difference between the FLDs predicted by isotropic hardening and those obtained with mixed hardening increases as $X_{\text {sat }}$ increases. One can also observe that kinematic hardening slightly decreases the level of the FLDs in the range of negative strain paths. However, this decrease is clearly more significant in the range of positive strain paths. The difference between the FLDs predicted by the two hardening models increases with the strain-path ratio $\rho$ (for $\rho \geq 0$ ). This difference is explained by the fact that the predictions based on kinematic hardening (mixed hardening in the present study) are rather similar to those given by a solid that develops a vertex on the yield surface. These observations are consistent with earlier studies (see, e.g. [30,32]).

\subsection{Relevance of the mixed hardening model: comparison with experiments}

This Section is dedicated to the comparison between the current numerical predictions and experimental results available in the literature. Such comparisons are necessary to assess the usefulness of the consideration of kinematic hardening in the theoretical modeling. In this aim, different results published in Graf and Hosford [41] and Graf and Hosford [42] will be analyzed. In these two papers, the effect of changing strain paths on the ductility limit of aluminum alloy 2008-T4 has been investigated by determining forming limit diagrams (FLDs) after prestraining. Sheets have been prestrained to several levels in uniaxial, biaxial, and plane-strain tension parallel and perpendicular to the prior rolling direction. In Graf and Hosford [41], the authors have compared the experimental results with some numerical predictions. To obtain their numerical predictions, the $\mathrm{M}-\mathrm{K}$ approach has been used and extended to include the effects of changing strain paths. Predictions incorporating abrupt strain path changes were in good agreement with the general trends observed experimentally, despite the fact that the predicted limit strains were found superior to the experimental ones. It must be recalled that only isotropic hardening has been considered in the theoretical M-K model developed in Graf and Hosford [41]. On the other hand, the plastic behavior has been modeled by the Hosford anisotropic yield criterion [43]. The experimental results, originally published in Graf and Hosford [41] and Graf and Hosford [42], have been compared with several numerical predictions [23,44,45]. All the above cited numerical contributions are based on the M-K approach to predict the formability limit. The main difference between the above cited numerical investigations is related to the modeling of the mechanical behavior of the studied sheets. Indeed, the Karafillis and Boyce yield function has been used in Cao et al. [23] and Yao and Cao [45] to model the plastic anisotropy, while the Hill yield function has been used in Nurcheshmeh and Green [44]. For the three above cited references, isotropic hardening has been considered and it has been modeled by the Swift hardening law. However, kinematic hardening has been taken into account only in Yao and Cao [45]. In this Section, comparisons between the current numerical predictions and the experimental results published in Graf and Hosford [41] are carried out. Unlike all the other Sections of the current paper, where the von Mises yield function is used, the Hosford anisotropic function is used as yield criterion in the current Section. This choice has been motivated by the fact that the Hosford yield function gives a more accurate description for the plastic anisotropy of aluminum alloy 2008-T4 [41]. Similar to the previous contributions $[23,41,45]$, our comparisons 


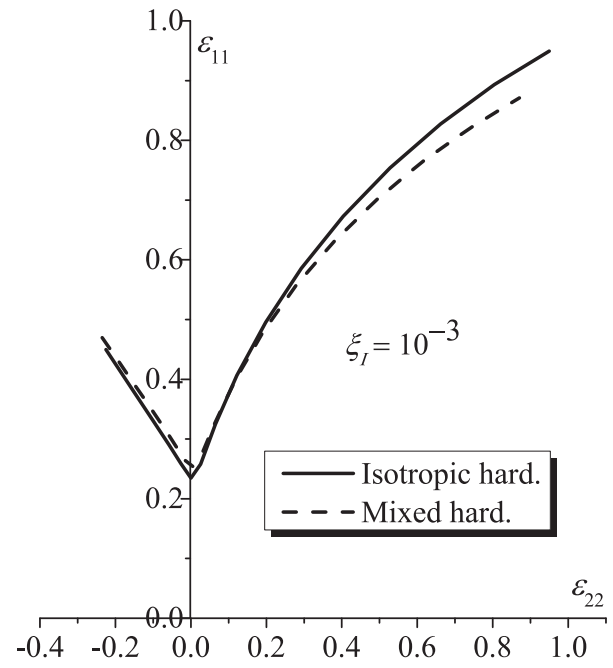

(a)

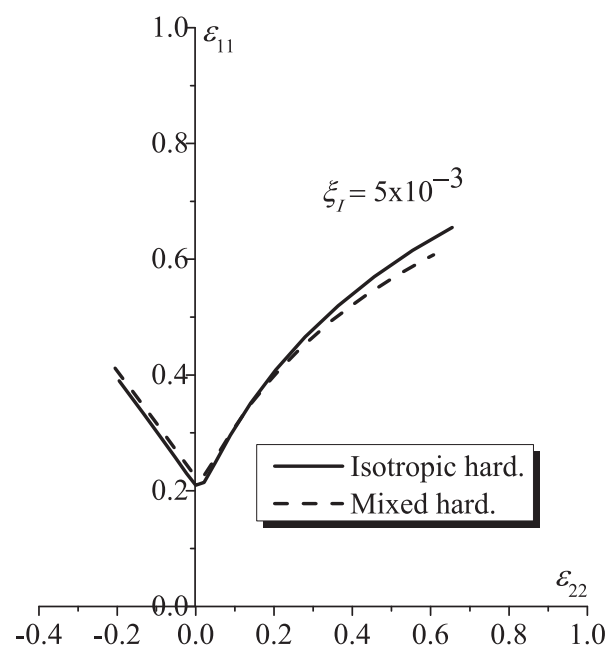

(c)

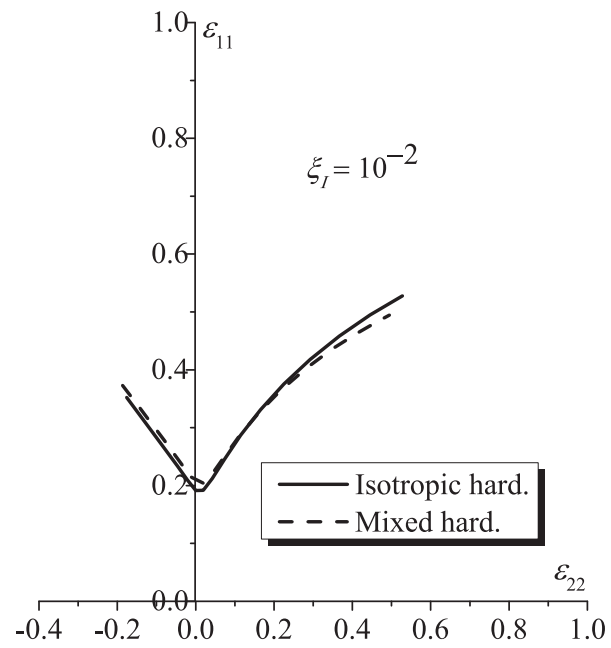

(e)

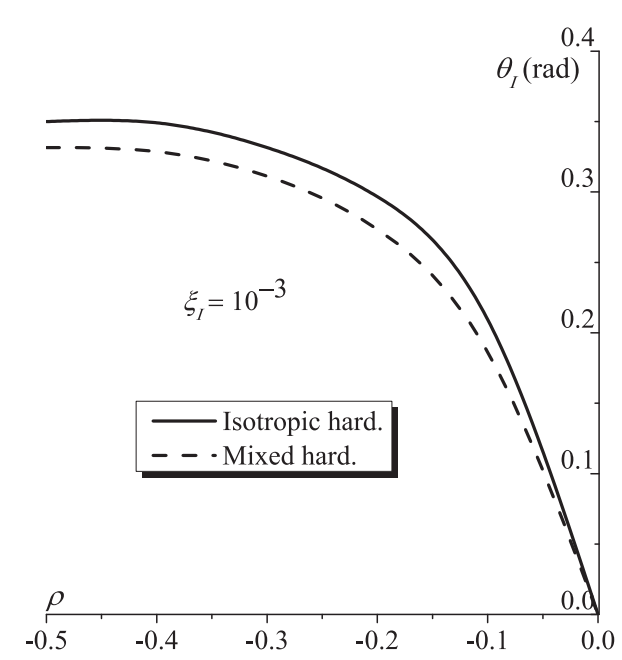

(b)

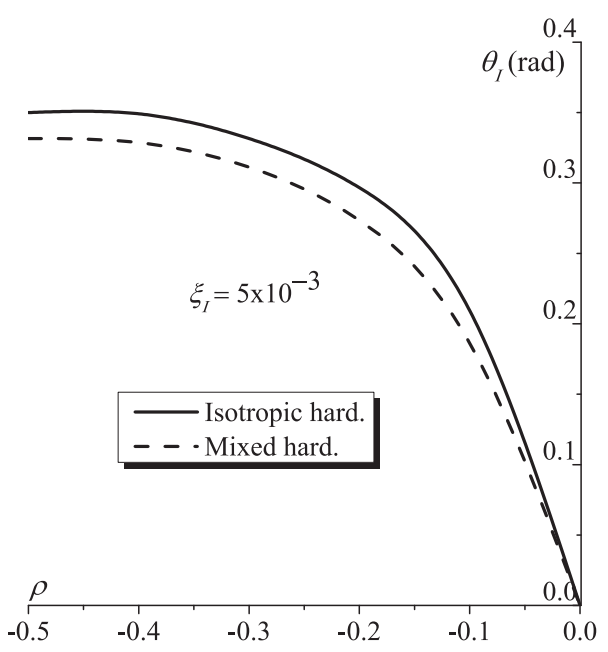

(d)

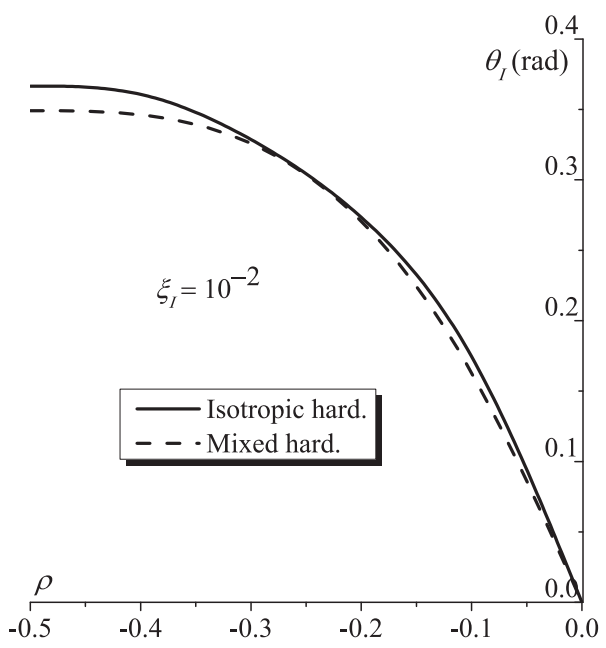

(f)

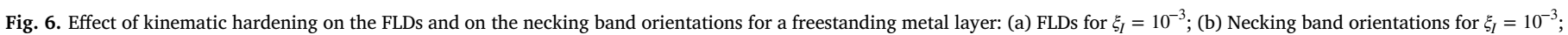
(c) FLDs for $\xi_{I}=5 \times 10^{-3}$; (d) Necking band orientations for $\xi_{I}=5 \times 10^{-3}$; (e) FLDs for $\xi_{I}=10^{-2}$; (f) Necking band orientations for $\xi_{I}=10^{-2}$. 
Table 2

Hardening parameters.

\begin{tabular}{|c|c|c|c|c|c|c|}
\hline & \multicolumn{2}{|c|}{ Isotropic hardening } & \multicolumn{4}{|c|}{ Mixed hardening } \\
\hline & $K(\mathrm{MPa})$ & $n$ & $K(\mathrm{MPa})$ & $n$ & $C_{x}$ & $X_{\text {sat }}(\mathrm{MPa})$ \\
\hline Material 1 & 589 & 0.3 & 447 & 0.221 & 2 & 100 \\
\hline Material 2 & 736 & 0.357 & 447 & 0.221 & 2 & 200 \\
\hline Material 3 & 886 & 0.402 & 447 & 0.221 & 2 & 300 \\
\hline Material 4 & 1039 & 0.437 & 447 & 0.221 & 2 & 400 \\
\hline
\end{tabular}

are restricted to the right-hand side of the forming limit diagrams (strain paths for which $\varepsilon_{2} \geq 0$ ). For such strain paths, the band remains normal to the direction of major strain during the loading, as has been demonstrated in Section 5.1. Consequently, the shearing components of the strain and stress tensors, both in the safe zone and the band, remain equal to 0 during the deformation. Under this condition and the planestress assumption, the yield criterion in both zones can be expressed by the following form (equivalent to Eq. (2)):

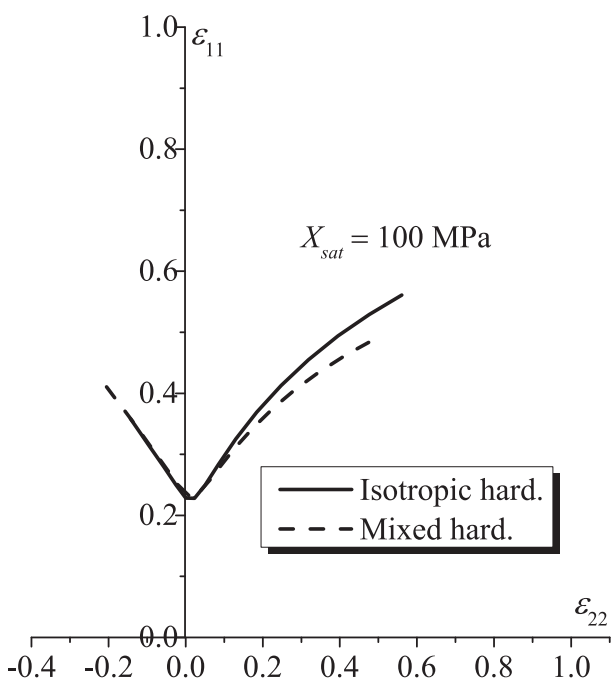

(a)

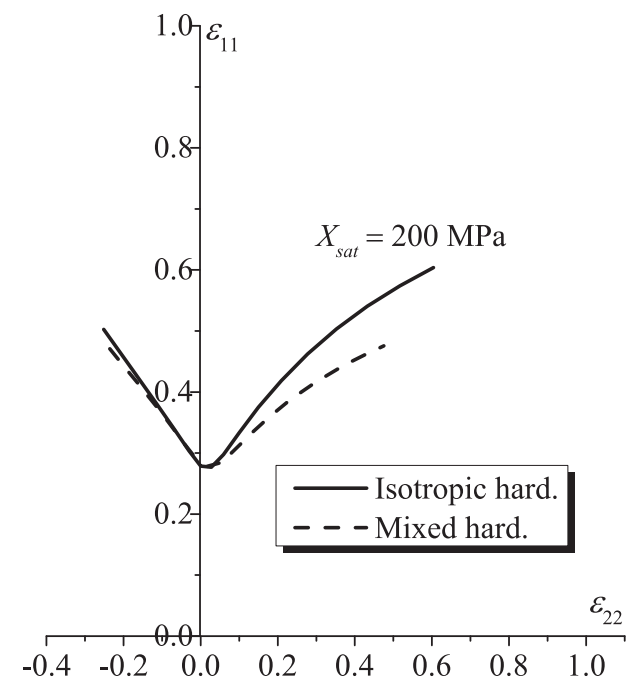

(c)
$F_{p}=\sqrt[a]{\frac{1}{(1+R)}\left(R\left(\tilde{S}_{22}-\widetilde{S}_{11}\right)^{a}+\left(2 \widetilde{S}_{11}+\widetilde{S}_{22}\right)^{a}+\left(\widetilde{S}_{11}+2 \widetilde{S}_{22}\right)^{a}\right)}-\sigma_{Y}=0$,

where $R$ is the Lankford coefficient and $a$ is a hardening exponent. As to $\tilde{S}_{11}$ and $\widetilde{S}_{22}$, they are defined by the following expressions:

$\tilde{S}_{11}=\sigma_{d 11}-X_{11} \quad ; \quad \tilde{S}_{22}=\sigma_{d 22}-X_{22}$,

where $\boldsymbol{\sigma}_{d}$ is the deviatoric part of the Cauchy stress tensor $\boldsymbol{\sigma}$, and $\mathbf{X}$ is the back-stress tensor.

Substituting $a=2$ recovers Hill's 1948 quadratic anisotropic yield function, and setting $R=1$ also gives rise to von Mises isotropic yield function. In the simulations presented in the current Section, coefficient $R$ is set to 0.78 and exponent $a$ is fixed to 8 (which is a typical value for FCC materials). The latter values have been originally provided in Graf and Hosford [41].

As previously explained, the objective of this section is to assess the usefulness of the consideration of kinematic hardening in the theoretical model. In this aim, simulations with isotropic and mixed hardening models are compared with the experimental results provided in Graf and Hosford [41]. For the isotropic hardening model, the hard-

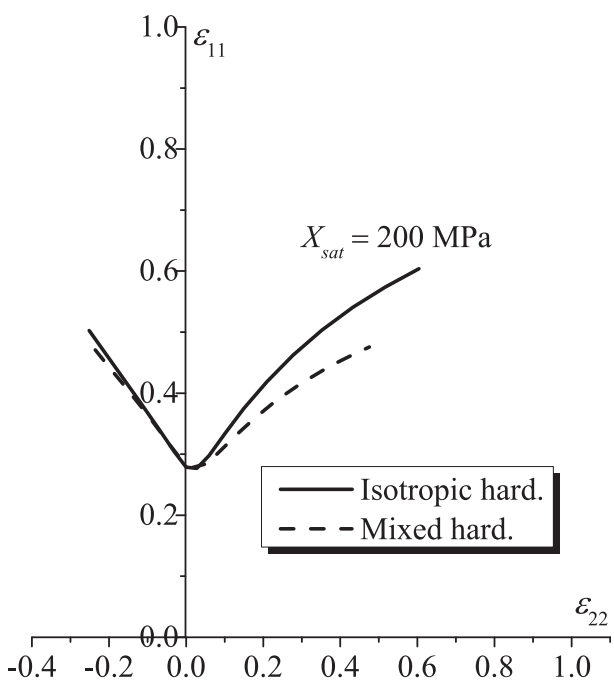

(b)

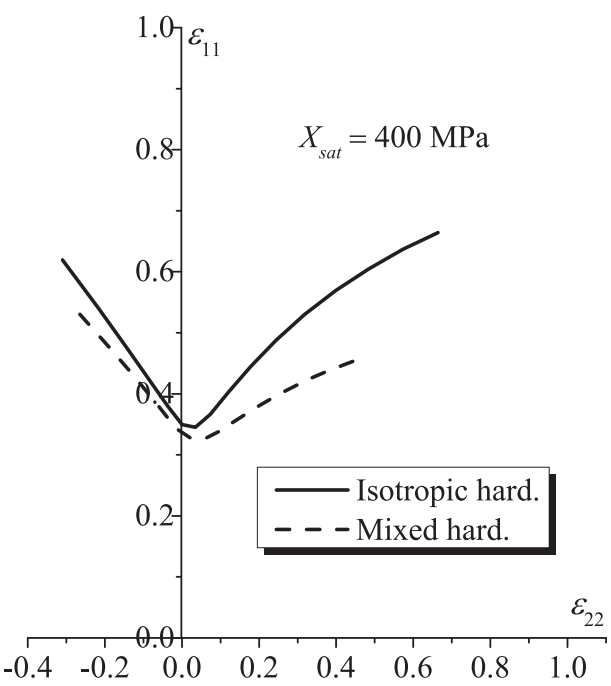

(d)

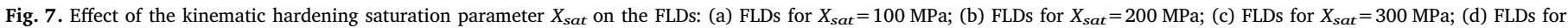
$X_{\text {sat }}=400 \mathrm{MPa}$. 
Table 3

Identified parameters for the aluminum alloy 2008-T4

\begin{tabular}{llllll}
\hline & $K(\mathrm{MPa})$ & $n$ & $C_{x}$ & $X_{\text {sat }}(\mathrm{MPa})$ & $\xi_{I}$ \\
\hline $\begin{array}{l}\text { Isotropic hardening } \\
\text { Mixed hardening }\end{array}$ & 530 & 0.25 & & & $8 \times 10^{-3}$ \\
& 400 & 0.2 & 90 & 2 & $15 \times 10^{-3}$ \\
\hline
\end{tabular}

ening parameters used in the simulations are the same as those given in Graf and Hosford [41]. These parameters have been identified by Graf and Hosford [41] on the basis of experimental stress-strain data obtained from monotonic uniaxial tensile loading. However, for the mixed hardening model, there are four parameters $\left(K, n, C_{x}\right.$ and $\left.X_{\text {sat }}\right)$ that need to be identified and, in this case, parameters $K$ and $n$ are likely to be different from their values fitted for the isotropic hardening model. It is also well known that monotonic experimental stress-strain curves are not sufficient to calibrate the parameters $\left(K, n, C_{x}\right.$ and $\left.X_{s a t}\right)$ of a mixed hardening model and, often, experimental stress-strain curves obtained after reverse or cyclic loading are required to obtain the optimal set for these four parameters. Unfortunately, cyclic data for this particular aluminum alloy is not available in the literature. To overcome this lack of experimental data, the experimental FLD of the as-received material (determined on the basis of linear strain paths and without any prestrain) will be used here as additional experimental fitting data, supplementary to the experimental monotonic stress-strain curve. Note that the same fitting technique has been previously followed in Cao et al. [11]. The value of the initial imperfection factor $\xi_{I}$ used in the M-K calculations is chosen to match the experimental FLD of the as-received material at the point of plane-strain tension $\left(\varepsilon_{2}=0\right)$. It should be noted that the identified value for $\xi_{I}$ is not the same for both hardening models (isotropic and mixed). The value of $\xi_{I}$ used for the isotropic hardening model is the same as that identified and used in Graf and Hosford [41]. The numerical values for all identified parameters are given in Table 3.

The comparisons between the predictions obtained with both hardening models are given in Fig. 8. The comparisons between the stress-strain curves are shown in Fig. 8(a). It is clearly revealed that the stress-strain curves obtained by both hardening models are almost the same. The different forming limit diagrams determined on the basis of linear strain paths are compared in Fig. 8(b). A detailed description for the different FLDs presented in Fig. 8(b) is given hereafter:

- FLD \# 1: the experimental FLD determined in Graf and Hosford [41] for the as-received aluminum alloy 2008-T4 for linear strain paths.

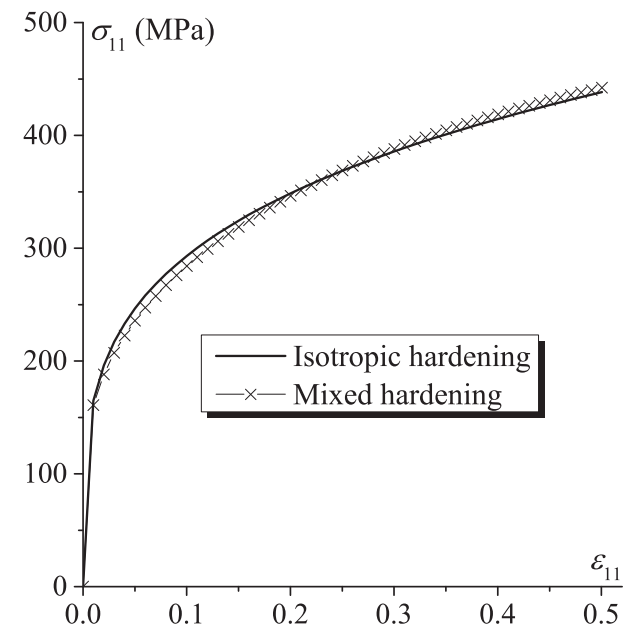

(a)
- FLD \# 2: the FLD calculated in Graf and Hosford [41]. It should be recalled that, in this numerical prediction, only isotropic hardening has been considered.

- FLD \# 3: the FLD predicted by the current numerical tool when hardening is assumed to be only isotropic.

- FLD \# 4: the FLD determined by the current numerical tool when hardening is assumed to be mixed (isotropic and kinematic).

It is clear that FLD \# 2 and FLD \# 3 match perfectly, which provides some additional validation for the developed numerical tool. The comparison between FLD \# 1 and FLD \# 4 shows that the mixed hardening parameters and the initial imperfection factor $\xi_{I}$ are correctly calibrated. Indeed, FLD \# 4 matches the experimental FLD (FLD \# 1) reasonably well. Furthermore, this comparison highlights the reliability and the flexibility of the mixed hardening model in the prediction of the forming limit diagrams, as compared to the isotropic hardening model. As demonstrated in Fig. 8(b), the use of the two hardening models (isotropic and mixed) leads to two distinct FLDs (FLD \# 3 and FLD \# 4), despite the high similarity between the associated stress-strain curves, as shown in Fig. 8(a). As commented in Section 5.1, this result can be explained by the fact that the strain path inside the band is non-linear during the deformation (contrary to the strain path in the homogeneous zone, which is assumed to be linear in this case). To further clarify this point, the evolution of the strain rate ratios $\dot{\varepsilon}_{22}^{S} / \dot{\varepsilon}_{11}^{S}$ and $\dot{\varepsilon}_{22}^{B} / \dot{\varepsilon}_{11}^{B}$ is plotted in Fig. 9 as a function of the strain component $\varepsilon_{11}^{S}$. Two particular strain paths are considered in this figure: $\rho=0.5$ and $\rho=1$. Although isotropic hardening is used for the predictions reported in Fig. 9, the corresponding conclusions are valid for the mixed hardening model as well. From Fig. 9, it is clear that the strain path ratio $\dot{\varepsilon}_{22}^{S} \dot{\varepsilon}_{11}^{S}$ is constant and equal to $\rho$ during the deformation. However, the strain path ratio $\dot{\varepsilon}_{22}^{B} / \dot{\varepsilon}_{11}^{B}$ evolves during the loading: it is almost equal to $\rho$ at the beginning of the loading, while it tends to 0 when plastic strain localization is approached. These results confirm the fact that the strain path in the band is non-linear during the deformation, even if it is linear in the safe (homogeneous) zone.

It is worth noting that, similar to the vast majority of $\mathrm{M}-\mathrm{K}$ approaches developed and used in the literature, the rupture stage is not modeled in the present $\mathrm{M}-\mathrm{K}$ approach. Accordingly, the localization of plastic deformation is adopted as the unique indicator for the determination of the formability limit.

In order to further analyze the relevance of the consideration of kinematic hardening in the mechanical modeling of aluminum alloy 2008-T4, abrupt strain path changes have been incorporated into the developed $\mathrm{M}-\mathrm{K}$ approach. This incorporation of sequential strain paths

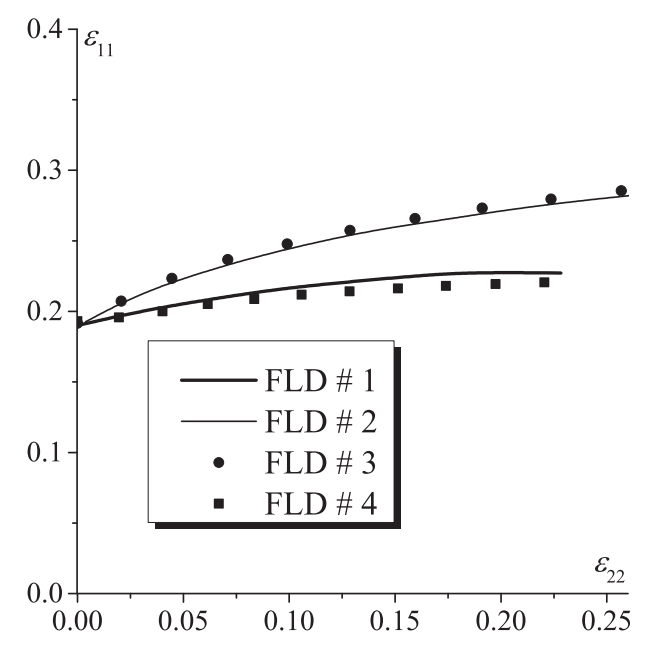

(b)

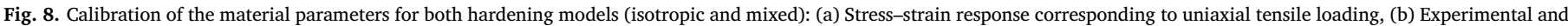
simulated FLDs. 


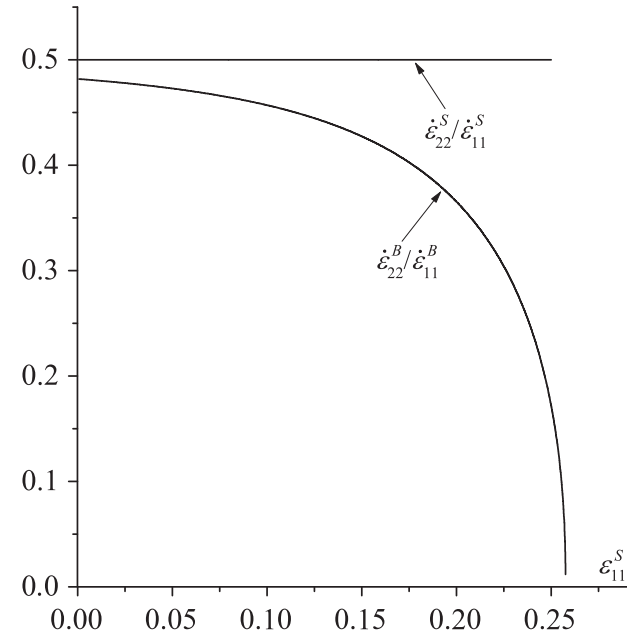

(a)

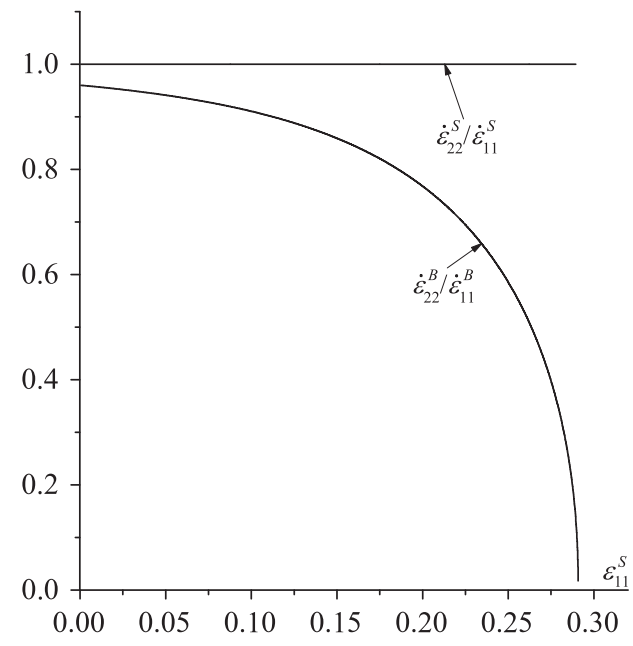

(b)

Fig. 9. Evolution of the strain rate ratios $\dot{\varepsilon}_{22}^{S} \mid \dot{\varepsilon}_{11}^{S}$ and $\dot{\varepsilon}_{22}^{B} \dot{\varepsilon}_{11}^{B}$ as a function of the strain component $\varepsilon_{11}^{S}$ (isotropic hardening model): (a) $\rho=0.5$, (b) $\rho=1$.

allowed us to simulate FLDs for bilinear strain paths. The algorithm given in Section 4 is used to simulate the prestrain stage. When the desired prestrain level is achieved, the current imperfection factor $\xi$ and the mechanical variables (equivalent strain, back-stress...) in the groove and the safe zone are used as starting values for the second (subsequent) strain path. Fig. 10 shows the comparisons between the experimental and the numerical FLDs, corresponding to different prestrain levels, for both plane-strain and equibiaxial tension states. The parameters used as input values for the different simulations reported in Fig. 10 are provided in Table 3. It must be recalled that the experimental monotonic uniaxial tensile stress-strain curve and the experimental FLD associated with linear strain paths have been used to calibrate the parameters of Table 3. As demonstrated in Fig. 10, the experimental results and the current numerical predictions using the mixed hardening model match very well qualitatively. Quantitatively, the results obtained with the mixed hardening model are much closer to experiments than those given by the isotropic hardening model. These results reveal that the mixed hardening model predicts more reliably the forming limits.

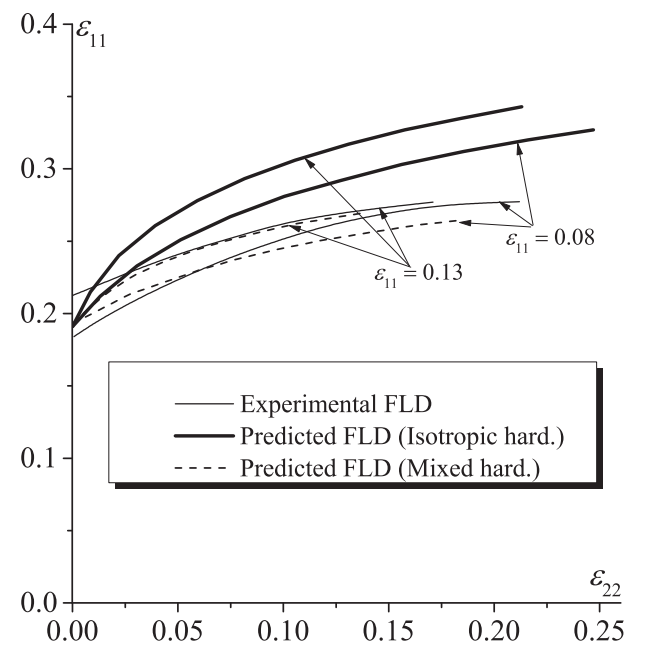

(a)

\subsection{Elastomer/metal bilayer}

In this section, a bilayer combination is considered, which consists of a metal layer supported by an elastomer substrate.

In order to emphasize the impact on the ductility of the bilayer of the different parameters involved (hardening parameters of the metal layer, relative thickness and shear modulus of the elastomer layer), a preliminary parametric study is first carried out. In this parametric study, attention is focused on the particular case of plane-strain tension $(\rho=0)$. This choice is motivated by the fact that, for the plane-strain tensile state, the strain path is proportional both in the band and in the safe zone. Hence, the incremental algorithm of Section 4 is no longer required, and a simpler compact analytical equation can be derived (Eq. (31)), which allows us to determine in a more straightforward way the limit strains. With this analytical equation we are also able to easily analyze the impact of the different parameters on the evolution of the limit strain. As previously stated, both hardening models (isotropic and mixed) provide the same limit strain in the case of the plane-strain tension loading path. Therefore, the effect of kinematic hardening on the limit strain cannot be highlighted in the current parametric study.

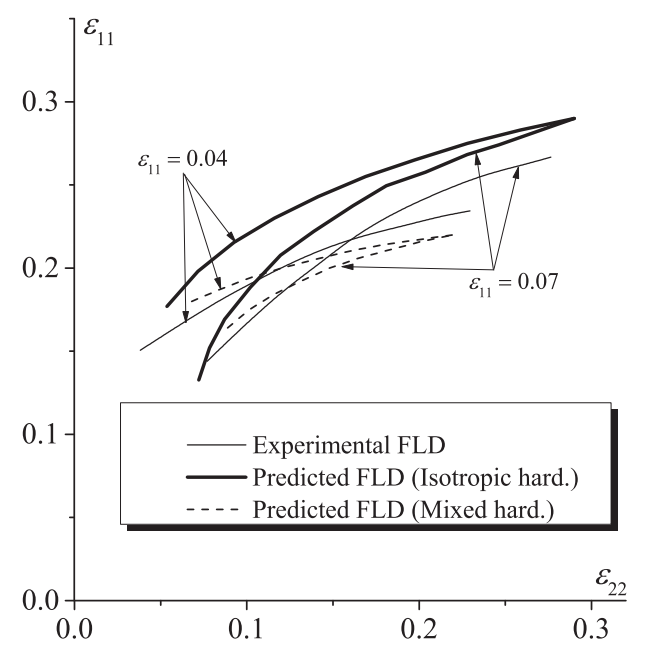

(b)

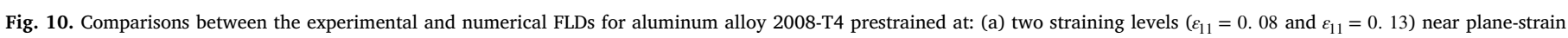
tension, (b) two straining levels $\left(\varepsilon_{11}=0.04\right.$ and $\left.\varepsilon_{11}=0.07\right)$ along equibiaxial tension. 
Nevertheless, this effect is extensively studied in the remainder of the paper, as already shown in Fig. 7, in particular (see also the associated discussions and conclusions). Hence, the current parametric study is restricted to the investigation of the impact on the limit strains of the following parameters: the isotropic hardening parameters $K$ and $n$ of the metal layer, the relative thickness and the shear modulus of the elastomer layer. As will be demonstrated in Fig. 13(c) and Fig. 13(d), the necking band orientation $\theta$ is equal to $0^{\circ}$ for the plane-strain tension loading path. Accordingly, the unit vector $\overrightarrow{\mathbf{n}}$ normal to the band is equal to $(1,0,0)$, and the equilibrium Eq. (15) simply reduces to the following scalar equation:

$h^{B} \sigma_{11}^{B}(M)+H^{B} \sigma_{11}^{B}(E)=h^{S} \sigma_{11}^{S}(M)+H^{S} \sigma_{11}^{S}(E)$.

By inserting Eq. (18) and their equivalent forms pertaining to the elastomer layer into Eq. (26), one obtains

$\left(h_{I}^{B} \sigma_{11}^{B}(M)+H_{I}^{B} \sigma_{11}^{B}(E)\right) e^{\varepsilon_{33}^{B}}=\left(h_{I}^{S} \sigma_{11}^{S}(M)+H_{I}^{S} \sigma_{11}^{S}(E)\right) e^{\varepsilon_{33}^{S}}$.

Taking into account the plane-strain state and the proportionality of the strain path both in the band and in the safe zone, one can easily deduce that $\varepsilon_{33}^{B}$ and $\varepsilon_{33}^{S}$ are equal to $-\varepsilon_{11}^{B}$ and $-\varepsilon_{11}^{S}$, respectively. As a result, Eq. (27) can be rewritten as

$\left(h_{I}^{B} \sigma_{11}^{B}(M)+H_{I}^{B} \sigma_{11}^{B}(E)\right) e^{-\varepsilon_{11}^{B}}=\left(h_{I}^{S} \sigma_{11}^{S}(M)+H_{I}^{S} \sigma_{11}^{S}(E)\right) e^{-\varepsilon_{11}^{S}}$.

On the other hand, the expressions of $\sigma_{11}^{B}(M), \sigma_{11}^{B}(E), \sigma_{11}^{S}(M)$ and $\sigma_{11}^{S}(E)$ can be obtained (after straightforward developments) by combining the plane-stress conditions and the constitutive equations of the metal and elastomer layers

$\sigma_{11}^{B}(M)=\left(\frac{2}{\sqrt{3}}\right)^{n+1} K\left(\varepsilon_{11}^{B}\right)^{n} \quad ; \quad \sigma_{11}^{S}(M)=\left(\frac{2}{\sqrt{3}}\right)^{n+1} K\left(\varepsilon_{11}^{S}\right)^{n}$.

$\sigma_{11}^{B}(E)=\mu\left(e^{2 \varepsilon_{11}^{B}}-e^{-2 \varepsilon_{11}^{B}}\right) \quad ; \quad \sigma_{11}^{S}(E)=\mu\left(e^{2 \varepsilon_{11}^{S}}-e^{-2 \varepsilon_{11}^{S}}\right)$

By inserting the above expressions into the equilibrium Eq. (28), one obtains

$e^{-\varepsilon_{11}^{B}}\left(h_{I}^{B}\left(\frac{2}{\sqrt{3}}\right)^{n+1} K\left(\varepsilon_{11}^{B}\right)^{n}+H_{I}^{B} \mu\left(e^{2 \varepsilon_{11}^{B}}-e^{-2 \varepsilon_{11}^{B}}\right)\right)$

$=e^{-\varepsilon_{11}^{S}}\left(h_{I}^{S}\left(\frac{2}{\sqrt{3}}\right)^{n+1} K\left(\varepsilon_{11}^{S}\right)^{n}+H_{I}^{S} \mu\left(e^{2 \varepsilon_{11}^{S}}-e^{-2 \varepsilon_{11}^{S}}\right)\right.$.

As previously stated, the initial imperfection is assumed to initiate within the metal layer and, accordingly, $H_{I}^{B}$ is equal to $H_{I}^{S}$. For the sake of simplicity, these two initial thicknesses will be designated as $H_{I}$, in what follows, and $h_{I}^{S}$ will be simply denoted $h_{I}$. By using the above notations and the definition (11) for the initial imperfection ratio $\xi_{l}$, Eq. (30) can be rewritten as

$e^{-\varepsilon_{11}^{B}}\left(\left(1-\xi_{I}\right)\left(\frac{2}{\sqrt{3}}\right)^{n+1} K\left(\varepsilon_{11}^{B}\right)^{n}+\left(\frac{H_{I}}{h_{I}}\right) \mu\left(e^{2 \varepsilon_{11}^{B}}-e^{-2 \varepsilon_{11}^{B}}\right)\right)$

$=e^{-\varepsilon_{11}^{S}}\left(\left(\frac{2}{\sqrt{3}}\right)^{n+1} K\left(\varepsilon_{11}^{S}\right)^{n}+\left(\frac{H_{I}}{h_{I}}\right) \mu\left(e^{2 \varepsilon_{11}^{S}}-e^{-2 \varepsilon_{11}^{S}}\right)\right.$.

To obtain the limit strain $\varepsilon_{11}$, Eq. (31) is incrementally solved. In this incremental procedure, the strain rate component $\dot{\varepsilon}_{11}^{B}$ is set to 1 (which means that $\varepsilon_{11}^{B}$ is equal to t), and time $\mathrm{t}$ is varied between 0 and 1 , with an increment size of $10^{-3}$. For each value of $\varepsilon_{11}^{B}$, Eq. (31) is solved by an iterative scheme, thus providing the corresponding value of $\varepsilon_{11}^{S}$. The limit strain is reached when $\dot{\varepsilon}_{11}^{B} / \dot{\varepsilon}_{11}^{S}$ becomes greater than 10 , as stated by Eq. (23). For all of the simulations in this parametric study, the initial imperfection ratio $\xi_{I}$ is set to $10^{-2}$. The results of this parametric study are reported in Fig. 11. On the basis of these results, the following conclusions can be drawn:

- The limit strains increase with the hardening exponent $n$, which is consistent with a number of previously reported experimental observations. Also, this result applies to both the freestanding metal layer and the substrate-supported metal layer.

- The hardening parameter $K$ has no impact on the limit strain for the freestanding metal layer. However, this parameter has a slight effect on the limit strain for the metal/elastomer bilayer. Indeed, in this latter case, the limit strain slightly decreases with $K$. By analyzing Eq. (31), this result is also expectable.

- The predicted limit strains increase with the shear modulus and/or the relative thickness of the elastomer layer. This means that the addition of an elastomer layer substantially retards the occurrence of necking, a result which is valid for both hardening models. This necking retardation is tied to the fact that under biaxial stretching, the incremental modulus of the elastomer layer remains essentially unchanged, or increases, while the incremental modulus of the metal layer steadily decreases. Additional details on this specific aspect will be provided in Appendix B.

In all calculations reported in the remainder of this section, the material parameters of the metal layer are those given in Table 1 . The shear modulus of the elastomer layer is set to $22 \mathrm{MPa}$. This latter choice is based on data for polyurea [46].

Fig. 12 reflects the effect of the elastomer layer and its initial thickness $H_{I}$, relative to that of the metal layer $h_{I}$, on the evolution of the ratio $\varepsilon_{11}^{B} / \varepsilon_{11}^{S}$ as a function of $\varepsilon_{11}^{S}$. It must be first noted that, in the case of uniaxial tension $(\rho=-0.5)$ with an initial inclination angle of $20^{\circ}$, localization is significantly retarded, due to the addition of the elastomer layer, for both initial imperfection factors $\left(5 \times 10^{-3}\right.$ and $10^{-2}$ ). In other words, in this special case of strain path and initial band orientation, the presence of the elastomer layer tends to enhance the immunity of the whole bilayer against necking. This phenomenon can be explained by the high resistance of the elastomer to necking and by the mechanical constraint applied by the substrate to the metal deformation. Because localization cannot be observed at a realistic strain level for $\rho=-0.5$ and $\theta_{I}=20^{\circ}$ (in this case, the limit strain exceeds 1), the curves in Fig. 12(a) and (b) are rather plotted for an alternative strain path $(\rho=-0.2)$, for which necking is observed. When the elastomer initial thickness $H_{I}$ is set to 0 (i.e., with no addition of elastomer substrate), the results of Fig. 3 are obviously consistently recovered. For both hardening models, the effect of the elastomer layer is to increase the critical strain $\varepsilon_{11}^{*}$ (which is the maximum value reached by $\varepsilon_{11}^{S}$ ). It is also observed that the dependence of the limit strain of the elastomer/metal bilayer on kinematic hardening is the same as for the freestanding metal layer. More specifically, when kinematic hardening is considered, the limit strain of the bilayer increases slightly for uniaxial tension and plane-strain tension, while it decreases more visibly in the case of equibiaxial tension. Also, the effect of the initial imperfection on the limit strain of the bilayer is quite similar to its effect in the case of a freestanding metal layer.

The combined effect of kinematic hardening and of the elastomer layer on the evolution of the critical strain $\varepsilon_{11}^{*}$ as a function of the initial band orientation $\theta_{I}$ is illustrated in Fig. 13. Similar to the case of a freestanding metal layer, we can conclude that, for the strain-path ratio $\rho=-0.3$, localization occurs along a slanted direction, lying between the two loading directions, as demonstrated in Fig. 13(a) and (b). It is also clear that the presence of the elastomer substrate reduces the value of the band inclination angle for this particular strain path, as shown in Fig. 13(a) and (b). However, this angle remains equal to 0 for positive strain paths $(\rho=0$ and $\rho=1)$, a result analogous to that found in the case of a freestanding metal layer (see Fig. 4). In the same way as before, for the equibiaxial tensile state, the critical strain $\varepsilon_{11}^{*}$ is found to be independent of the value of $\theta_{I}$ (see Fig. 13(e) and (f)). However, this critical strain obviously depends on the material parameters of both layers, on the thickness ratio $H_{I} / h_{I}$ and on the initial imperfection factor $\xi_{I}$. On the whole, Fig. 13 reveals that the effect of kinematic hardening on the limit strain of the bilayer follows the same trends 


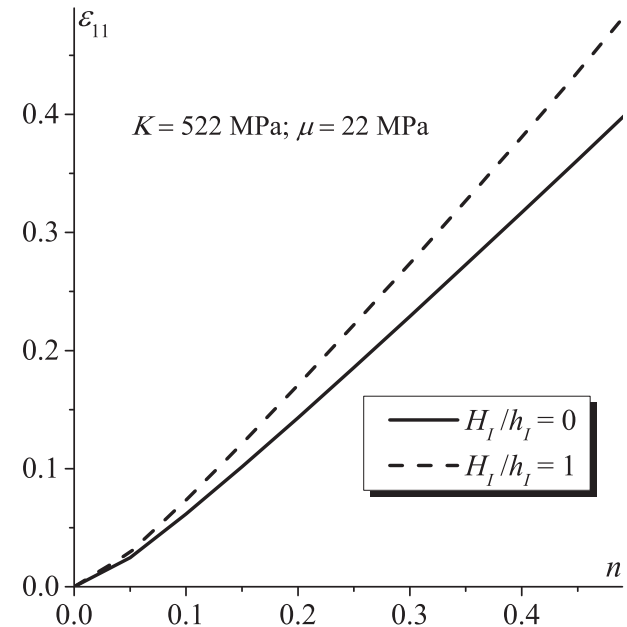

(a)

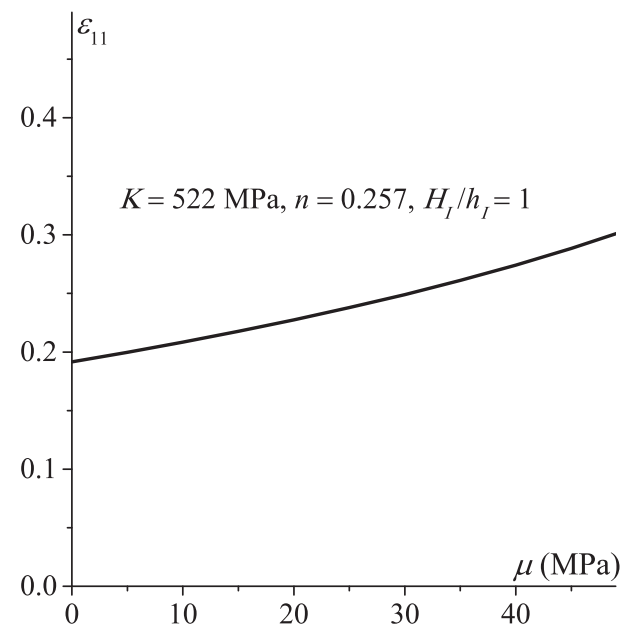

(c)

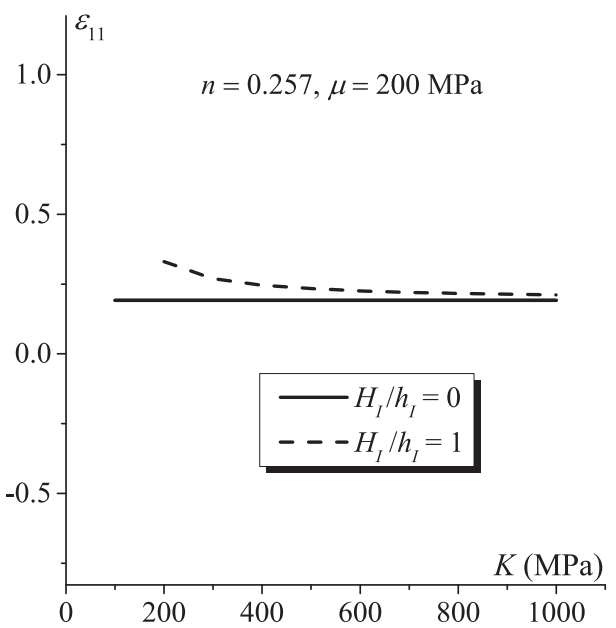

(b)

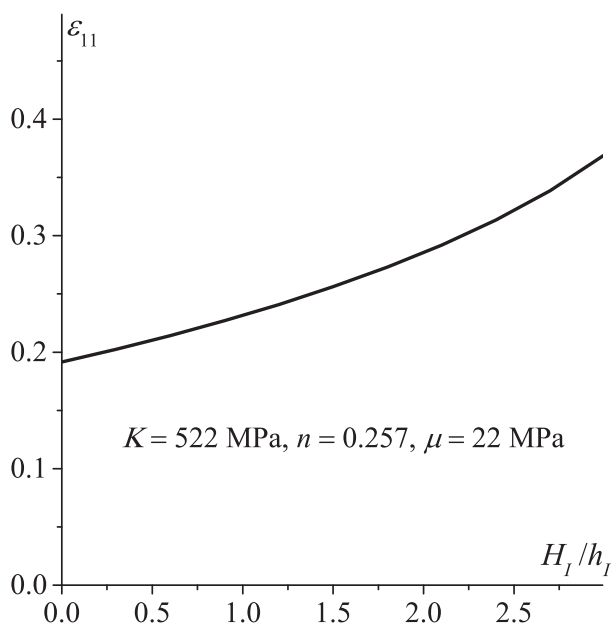

(d)

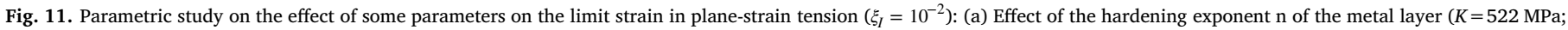

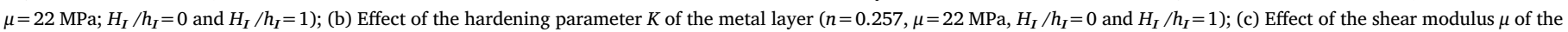
elastomer ( $\left.K=522 \mathrm{MPa}, n=0.257, H_{I} / h_{I}=1\right)$; (d) Effect of the thickness ratio $H_{I} / h_{I}(K=522 \mathrm{MPa}, n=0.257, \mu=22 \mathrm{MPa})$.

observed in Fig. 4 for a freestanding metal layer.

In Fig. 14, the combined effect of kinematic hardening and of the elastomer layer on the FLDs and on the necking band orientations is illustrated for three initial imperfection factors, namely, $10^{-3}, 5 \times 10^{-3}$ and $10^{-2}$. Fig. 14(a), (c) and (e) confirm the previous observations reflected by Fig. 12 and Fig. 13; namely, the elastomer layer substantially enhances the ductility of the bilayer for both hardening models and all of the strain paths. Also, we can conclude that the impact of the addition of an elastomer substrate is more significant than the effect of kinematic hardening. The evolution of the necking band orientation, as a function of the strain-path ratio (for negative values of the latter), is plotted in Fig. 14(b), (d) and (f). These figures confirm the trends reflected by Fig. 13(a) and (b); namely, the elastomer substrate reduces the value of the band inclination.

Additional simulations have been carried out in order to further investigate the effect of the relative thickness of the elastomer layer on the ductility limit of the whole bilayer. The associated results are reported in Fig. 15, for an initial imperfection factor $\xi_{I}$ equal to $10^{-2}$. From this figure, it can be seen that the larger the relative thickness $H_{I}$ $/ h_{I}$, the more significant the retardation of necking occurrence. These results clearly demonstrate the significant impact of the elastomer substrate on the ductility of the bilayer, and confirm the previous discussions on the positive effect of the elastomer layer on the necking retardation. Moreover, these conclusions are valid for both hardening models.

\section{Conclusions}

The forming limits for freestanding metal layers and substratesupported metal layers have been numerically determined using both isotropic and mixed hardening models. The conclusions based upon the present work are given as follows:

- Overall, the predicted limit strains for the whole FLD tend to decrease when the saturation parameter of the kinematic hardening model increases, as illustrated in Fig. 7. For small to moderate values of the kinematic hardening saturation parameter, the difference between the FLDs predicted by the isotropic hardening model and those determined by the mixed hardening model is not very significant.

- As demonstrated in Fig. 10, the experimental results and the numerical predictions based on the mixed hardening model match very well qualitatively. Quantitatively, the results obtained with the mixed hardening model are much closer to experiments than those given by the isotropic hardening model. These results reveal that the mixed hardening model predicts more reliably the forming limits. 


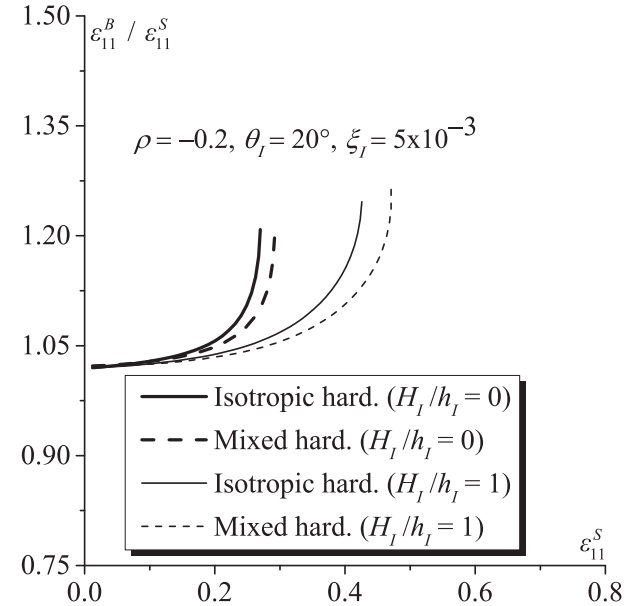

(a)

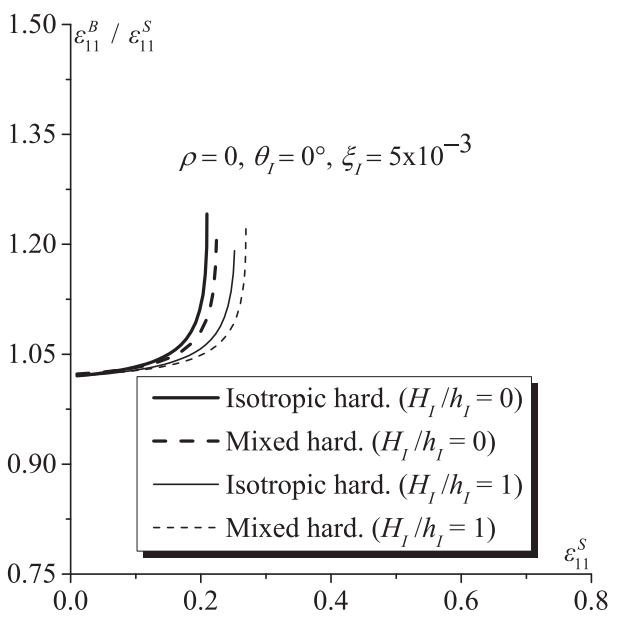

(c)

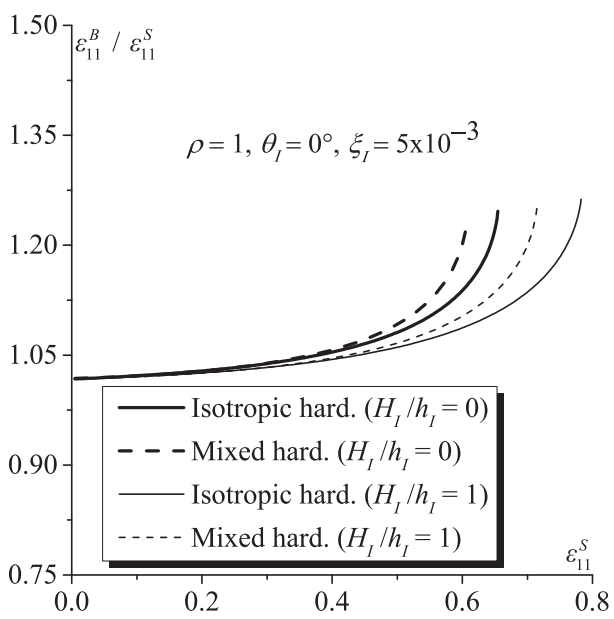

(e)

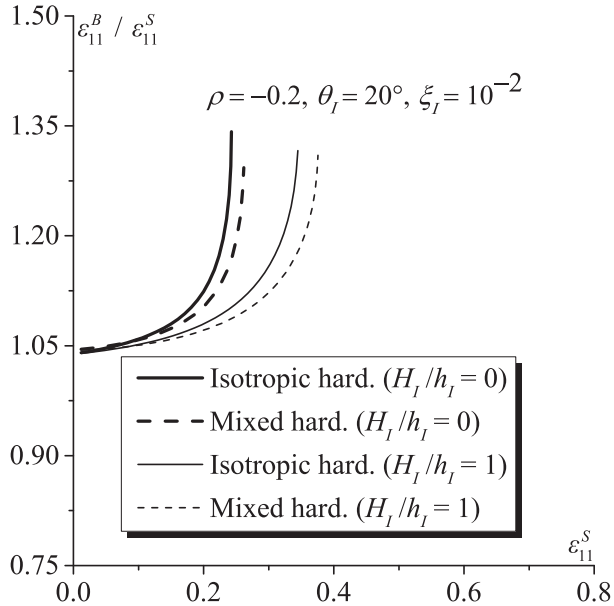

(b)

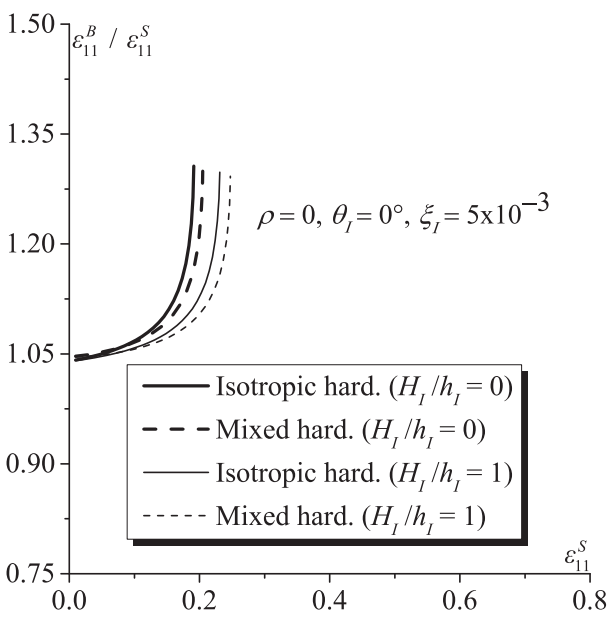

(d)

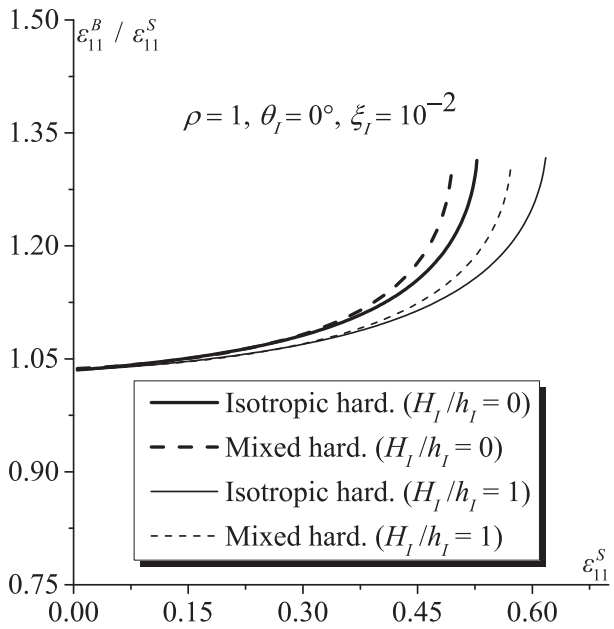

(f)

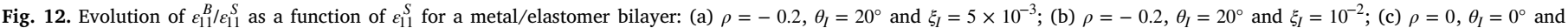
$\xi_{I}=5 \times 10^{-3}$; (d) $\rho=0, \theta_{I}=0^{\circ}$ and $\xi_{I}=10^{-2}$; (e) $\rho=1, \theta_{I}=0^{\circ}$ and $\xi_{I}=5 \times 10^{-3}$; (f) $\rho=1, \theta_{I}=0^{\circ}$ and $\xi_{I}=10^{-2}$.

- For both hardening models, the addition of an elastomer layer substantially enhances the ductility limit of the resulting metal/ elastomer bilayer. This neck retardation is attributable, on the one hand, to the high resistance of the elastomer material to necking and, on the other hand, to the mechanical constraint of the elastomer substrate to the metal deformation.

- In both cases of freestanding metal layers and metal/elastomer bilayers, the necking band for positive strain paths $(\rho \geq 0)$ is found to be perpendicular to the direction of major strain. Also, for equibiaxial tension $(\rho=1)$, the value of the critical strain is independent of 


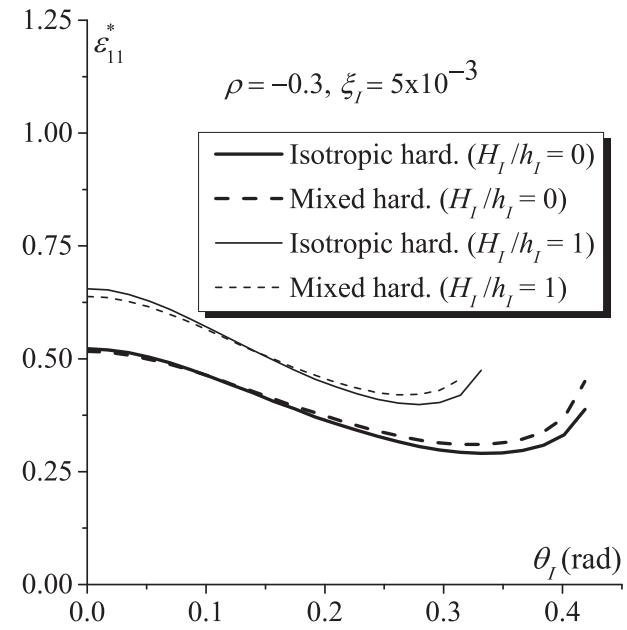

(a)

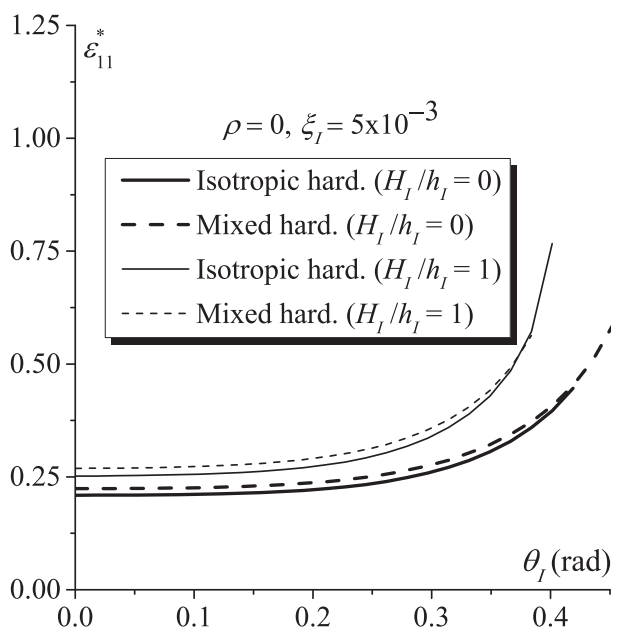

(c)

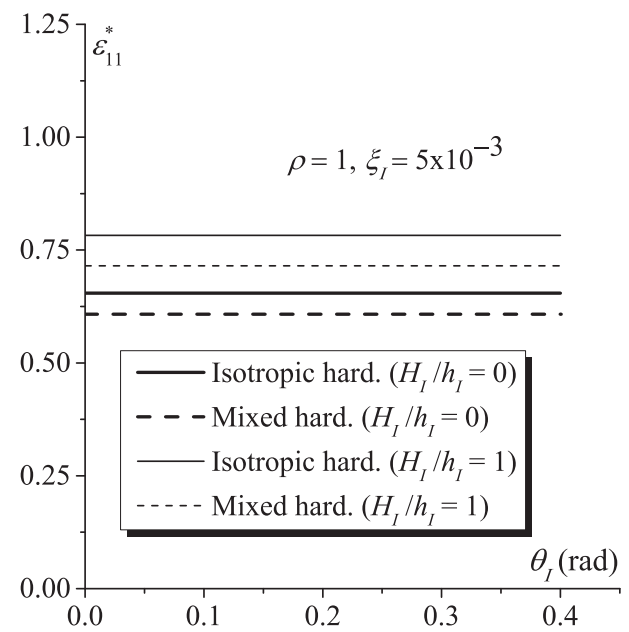

(e)

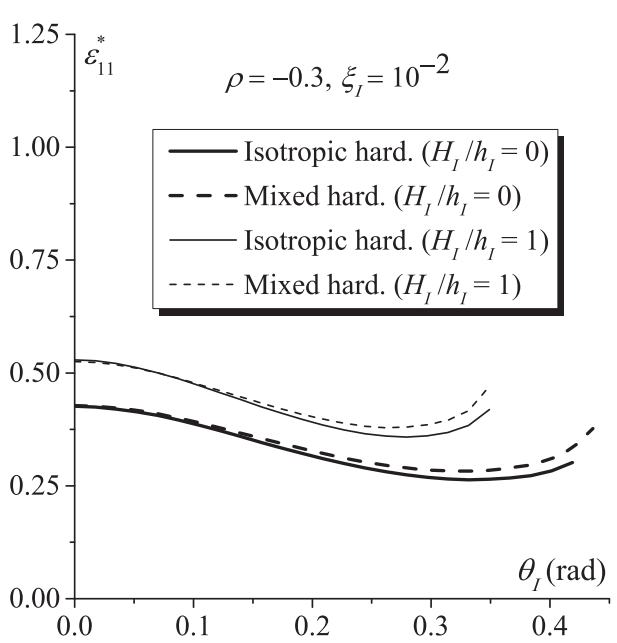

(b)

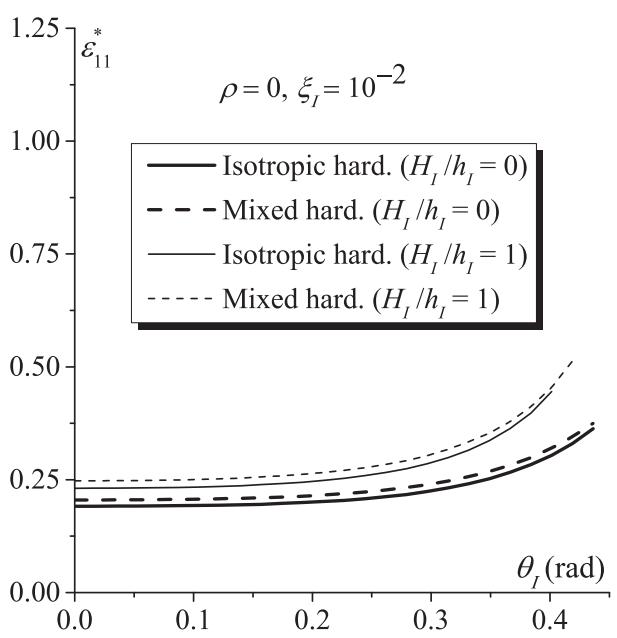

(d)

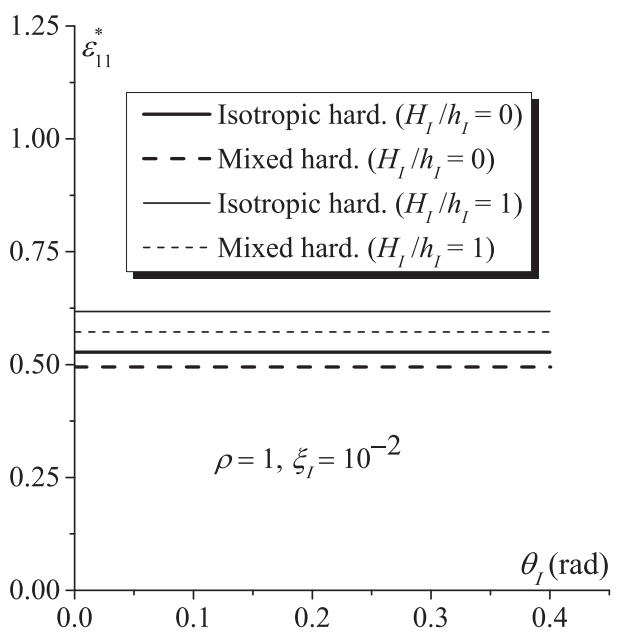

(f)

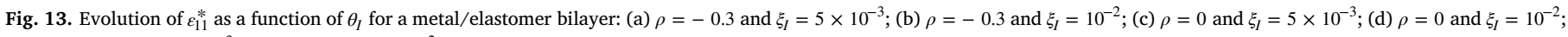
(e) $\rho=1$ and $\xi_{I}=5 \times 10^{-3}$; (f) $\rho=1$ and $\xi_{I}=10^{-2}$. 


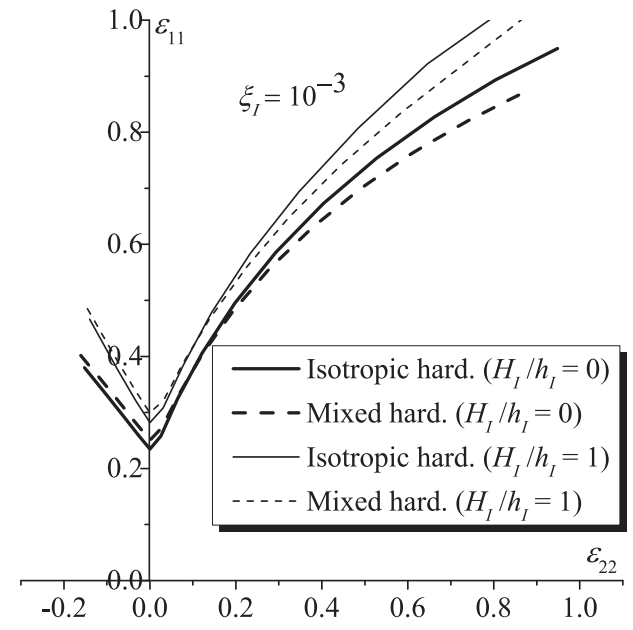

(a)

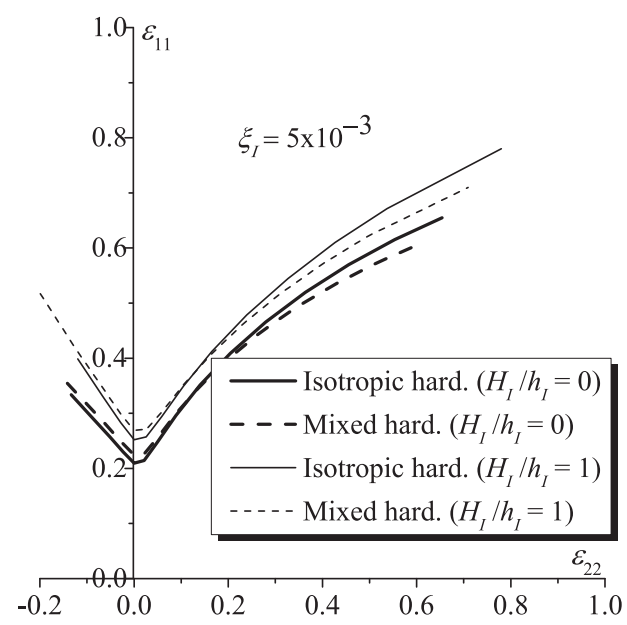

(c)

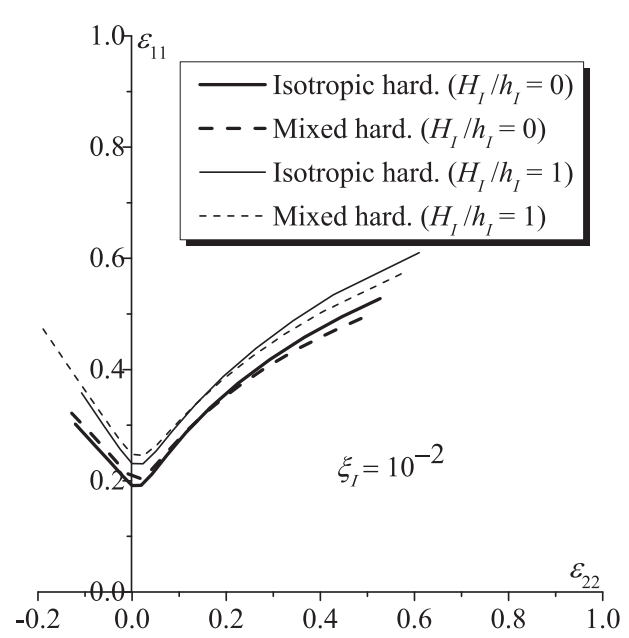

(e)

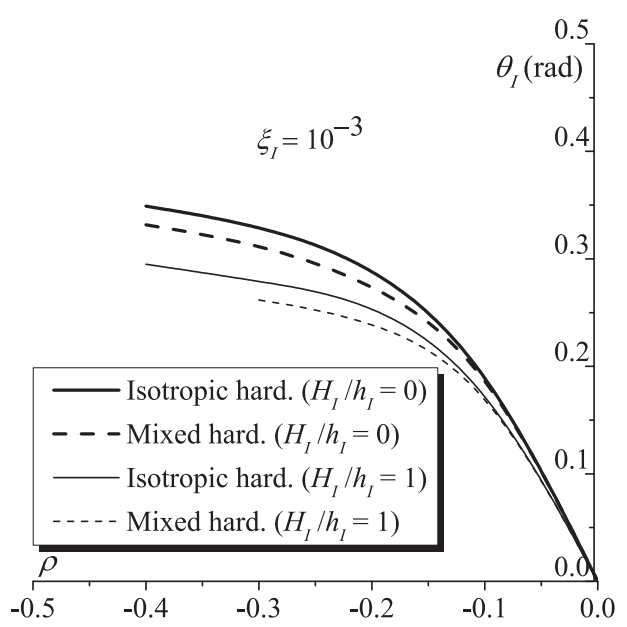

(b)

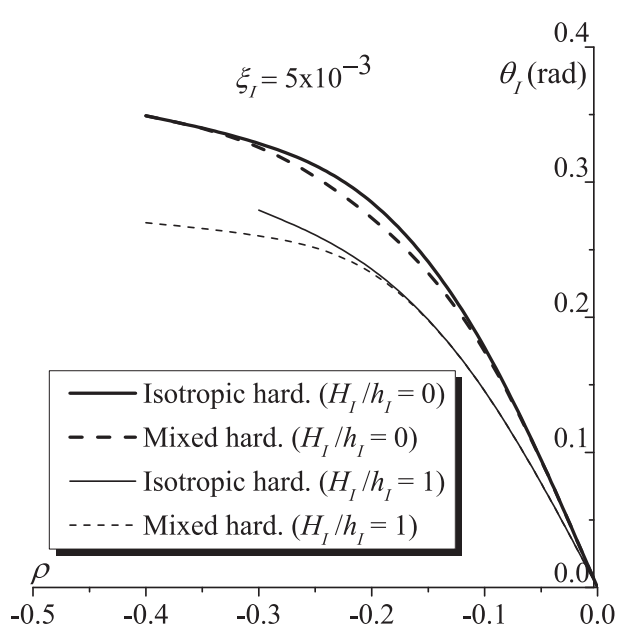

(d)

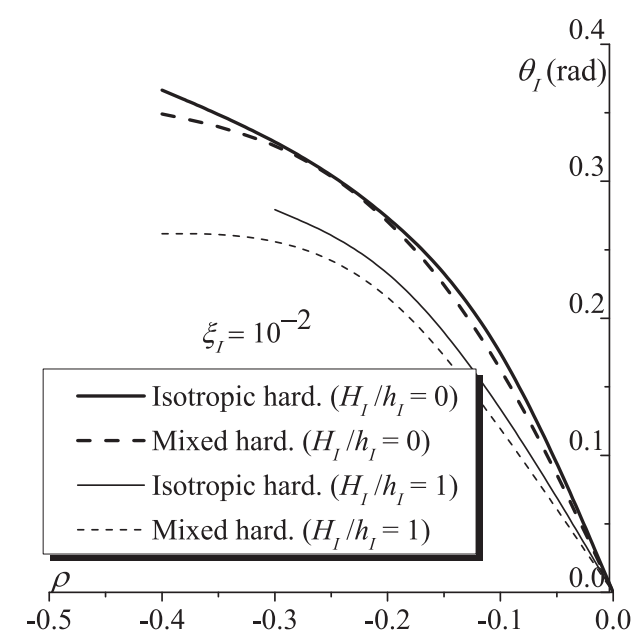

(f)

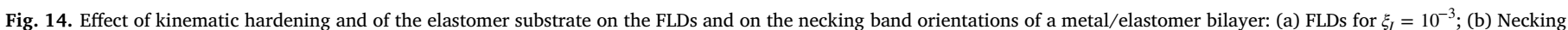
band orientations for $\xi_{I}=10^{-3}$; (c) FLDs for $\xi_{I}=5 \times 10^{-3}$; (d) Necking band orientations for $\xi_{I}=5 \times 10^{-3}$; (e) FLDs for $\xi_{I}=10^{-2}$; (f) Necking band orientations for $\xi_{I}=10^{-2}$. 


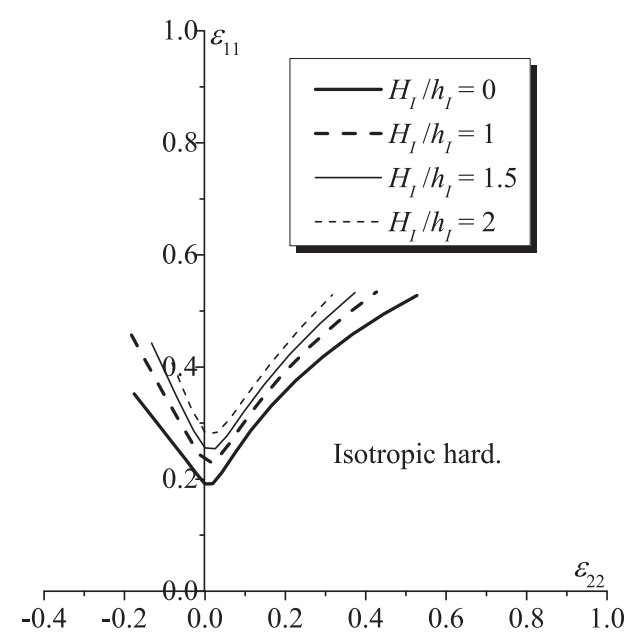

(a)

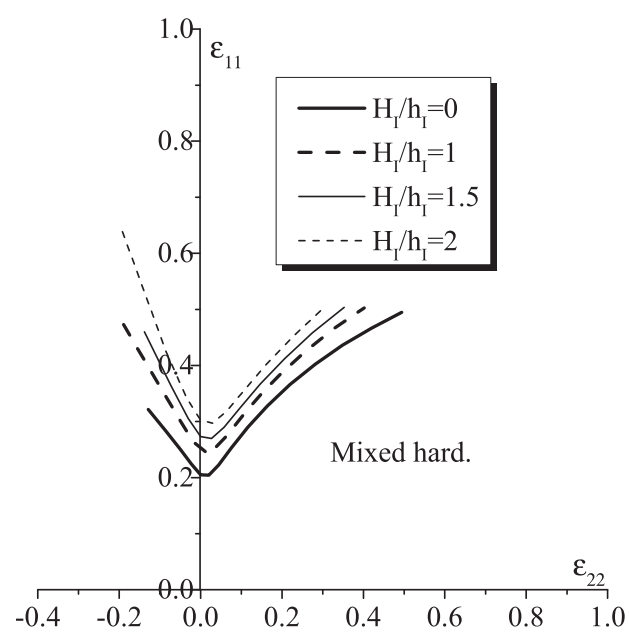

(b)

Fig. 15. Effect of the elastomer relative thickness $H_{I} / h_{I}$ on the predicted FLDs: (a) Isotropic hardening; (b) Mixed hardening.

the initial groove orientation. These results are valid for both hardening models, and are fully consistent with previous studies from the literature.

\section{Appendix A. Incremental algorithm}

The aim of this incremental algorithm is to integrate the governing equations for the M-K analysis over a typical time increment $\left[t_{n}, t_{n+1}\right]$. For this purpose, we assume that besides the material parameters, the following quantities are known at $t_{n}$ in both layers:

- $\mathbf{F}_{t_{n}}^{B}$ (resp. $\mathbf{F}_{t_{n}}^{S}$ ): the deformation gradient in the band (resp. in the safe zone).

- $\varepsilon_{e q t_{n}}^{B}$ (reps. $\varepsilon_{e q t_{n}}^{S}$ ): the equivalent strain in the band (resp. in the safe zone).

- $\mathbf{X}_{t_{n}}^{B}$ (resp. $\mathbf{X}_{t_{n}}^{S}$ ): the back-stress tensor corresponding to the metal layer in the band (resp. in the safe zone). layer.

It must be recalled that, due to perfect adherence assumption (12), $\mathbf{F}_{t_{n}}^{B}, \mathbf{F}_{t_{n}}^{S}, \boldsymbol{\varepsilon}_{\text {eq }}^{B}, \boldsymbol{\varepsilon}_{\text {eq } t_{n}}^{S}$ take the same values both in the metal and in the elastomer

In order to simplify notations, subscript $t_{n+1}$, indicating that the corresponding quantity is expressed at $t_{n+1}$, will be omitted hereinafter, with the implied understanding that this quantity is evaluated at $t_{n+1}$, unless otherwise indicated.

As any numerical algorithm for the time integration of mechanical constitutive equations, which are assumed to be strain-driven, a prescribed quantity must be chosen as a loading parameter over $\left[t_{n}, t_{n+1}\right]$. In order to simplify the computation and to avoid some numerical problems, this prescribed quantity should be chosen among the different strain quantities that evolve monotonically before and after the occurrence of localization (the onset of localization being not a priori known). Regarding the strain quantities in the safe zone, these evolve monotonically before strain localization, but remain constant afterwards (as the behavior is taken rigid-plastic, thus precluding elastic unloading in the safe zone following strain localization). Consequently, it is more suitable to choose the prescribed quantity among the velocity gradient components in the band. In our case, the component $G_{33}^{B}$ is chosen as prescribed quantity over $\left[\begin{array}{ll}t_{n} & t_{n+1}\end{array}\right]$. The mechanical behavior in both layers being taken strain-rate independent, the prescribed value for $G_{33}^{B}$ may be chosen quite freely without influencing the predicted necking limit. For convenience, $G_{33}^{B}$ is assumed to be constant and equal to -1 . This latter choice in conjunction with Eq. (22) leads to the following relation (where the different variables are expressed at $t_{n+1}$ ):

$(1+\rho) \dot{\varepsilon}_{11}^{S}+\dot{c}_{1} n_{1}+\dot{c}_{2} n_{2}=1$.

The unit normal vector $\overrightarrow{\mathbf{n}}$ is equal to ( $\cos \theta, \sin \theta, 0$ ), and hence $\overrightarrow{\mathbf{n}}$ only depends on $\theta$. On the other hand, $\theta$ can be expressed as follows (an equation equivalent to Eq. (16)):

$\operatorname{Tan}(\theta)=e^{(1-\rho)} \int_{0}^{t_{n+1}} \dot{\varepsilon}_{11}^{S} d t \operatorname{Tan}\left(\theta_{I}\right)$

which can also be rewritten in the following incremental form:

$\operatorname{Tan}(\theta)=e^{\Delta t(1-\rho) \dot{\varepsilon}_{11}^{S}} \operatorname{Tan}\left(\theta_{t_{n}}\right)$,

where $\theta_{t_{n}}$ is the orientation of the band at the beginning of the time increment, which is assumed to be known in this incremental formulation.

Accordingly, for a given strain path $\rho$ and initial orientation $\theta_{I}$ (as explained in the algorithm of Section 4), Eq. (A.1) depends on three scalar unknowns: $\dot{\varepsilon}_{11}^{S}, \dot{c}_{1}$ and $\dot{c}_{2}$.

The back-stress $\mathbf{X}$ can be integrated by an implicit scheme over $\left[\begin{array}{ll}t_{n}, & t_{n+1}\end{array}\right]$

$\mathbf{X}=\mathbf{X}_{t_{n}}+\Delta t \dot{\mathbf{X}}$.

Introducing the equality (A.4) into Eq. (4) leads to

$\dot{\mathbf{X}}=C_{x}\left(X_{s a t} \dot{\boldsymbol{\varepsilon}}-\dot{\varepsilon}_{e q}\left(\mathbf{X}_{t_{n}}+\Delta t \dot{\mathbf{X}}\right)\right)$. 
By rearranging Eq. (A.5), we can obtain the following expression for $\dot{\mathbf{X}}$ :

$\dot{\mathbf{X}}=\frac{C_{x}\left(X_{s a t} \dot{\varepsilon}-\mathbf{X}_{t_{n}} \dot{\varepsilon}_{e q}\right)}{1+\Delta t C_{x} \dot{\varepsilon}_{e q}}$

The back-stress $\mathbf{X}$ at $t_{n+1}$ can then be expressed in the following form:

$\mathbf{X}=\mathbf{X}_{t_{n}}+\frac{\Delta t C_{x}\left(X_{s a t} \dot{\varepsilon}-\mathbf{X}_{t_{n}} \dot{\varepsilon}_{e q}\right)}{1+\Delta t C_{x} \dot{\varepsilon}_{e q}}$.

Finally, the back-stress within the metal layer both in the safe zone and in the band, noted respectively $\mathbf{X}^{S}$ and $\mathbf{X}^{B}$, can be expressed as follows:

$\mathbf{X}^{S}=\mathbf{X}_{t_{n}}^{S}+\frac{\Delta t C_{x}\left(X_{s a t} \dot{\varepsilon}^{S}-\mathbf{X}_{t_{n}}^{S} \dot{\varepsilon}_{e q}^{S}\right)}{1+\Delta t C_{x} \dot{\varepsilon}_{e q}^{S}} \quad ; \quad \mathbf{X}^{B}=\mathbf{X}_{t_{n}}^{B}+\frac{\Delta t C_{x}\left(X_{s a t} \dot{\boldsymbol{\varepsilon}}^{B}-\mathbf{X}_{t_{n}}^{B} \dot{\varepsilon}_{e q}^{B}\right)}{1+\Delta t C_{x} \dot{\varepsilon}_{e q}^{B}}$.

Considering expression (21) (resp. (22)) pertaining to $\mathbf{G}^{S}$ (resp. $\mathbf{G}^{B}$ ), we can easily observe that $\dot{\boldsymbol{\varepsilon}}^{S}$ (resp. $\dot{\boldsymbol{\varepsilon}}^{B}$ ), which is the symmetric part of $\mathbf{G}^{S}$ (resp. $\mathbf{G}^{B}$ ), is function only of the unknown $\dot{\varepsilon}_{11}^{S}$ (resp. the unknowns $\dot{\varepsilon}_{11}^{S}, \dot{c}_{1}$ and $\dot{c}_{2}$ ). Accordingly, $\mathbf{X}^{S}$ and $\mathbf{X}^{B}$ are completely determined once $\dot{\varepsilon}_{11}^{S}, \dot{c}_{1}$ and $\dot{c}_{2}$ are known.

In the same way, Eq. (6) reveals that $\boldsymbol{\sigma}_{d}^{S}(M)$ (resp. $\boldsymbol{\sigma}_{d}^{B}(M)$ ) is function of the unique unknown $\dot{\varepsilon}_{11}^{S}$ (resp. the three unknowns $\dot{\varepsilon}_{11}^{S}, \dot{c}_{1}$ and $\dot{c}_{2}$ ).

On the other hand, the velocity gradients $\mathbf{F}^{S}$ and $\mathbf{F}^{B}$ can be expressed as functions of $\mathbf{G}^{S}$ and $\mathbf{G}^{B}$, respectively

$\mathbf{F}^{S}=e^{\Delta t \mathbf{G}^{S}} \mathbf{F}_{t_{n}}^{S} ; \quad \mathbf{F}^{B}=e^{\Delta t \mathbf{G}^{B}} \mathbf{F}_{t_{n}}^{B}$.

Accordingly, $\mathbf{F}^{S}$ and $\mathbf{F}^{B}$ (and hence the associated left Cauchy-Green tensors $\mathbf{V}^{S}$ and $\mathbf{V}^{B}$ ) depend only on the three unknowns $\dot{\varepsilon}_{11}^{S}, \dot{c}_{1}$ and $\dot{c}_{2}$. By analyzing the constitutive Eq. (7) for the elastomer, we can conclude that the computation of $\dot{\varepsilon}_{11}^{S}, \dot{c}_{1}$ and $\dot{c}_{2}$ allows us to completely determine the deviatoric stresses $\mathbf{B}^{S}(E)$ and $\mathbf{B}^{B}(E)$.

Also, the current thicknesses of the metal and elastomer layers, both in the safe zone and in the band, can be written in the following incremental forms:

$h^{S}=h_{t_{n}}^{S} e^{\Delta t G_{33}^{S}} \quad ; \quad h^{B}=h_{t_{n}}^{B} e^{\Delta t G_{33}^{B}} \quad ; \quad H^{S}=H_{t_{n}}^{S} e^{\Delta t G_{33}^{S}} \quad ; \quad H^{B}=H_{t_{n}}^{B} e^{\Delta t G_{33}^{B}}$,

which reveals that $h^{S}, h^{B}, H^{S}, H^{B}$ depend only on the three unknowns $\dot{\varepsilon}_{11}^{S}, \dot{c}_{1}$ and $\dot{c}_{2}$.

The equilibrium Eq. (15) can be expressed in the following index form:

$\forall i=1,2,3: \quad\left(h^{B} \sigma_{i j}^{B}(M)+H^{B} \sigma_{i j}^{B}(E)\right) \cdot n_{j}=\left(h^{S} \sigma_{i j}^{S}(M)+H^{S} \sigma_{i j}^{S}(E)\right) \cdot n_{j} \quad ; \quad j=1,2,3$.

The normal vector being lying in the plane of the sheet, as shown in Fig. 1, its third component $n_{3}$ is equal to 0 . Furthermore, by virtue of the plane-stress conditions, $\sigma_{i 3}^{B}$ and $\sigma_{i 3}^{S}$ are equal to 0 . Therefore, Eq. (A.11) can be reduced to the in-plane components

$\forall i=1,2: \quad\left(h^{B} \sigma_{i j}^{B}(M)+H^{B} \sigma_{i j}^{B}(E)\right) \cdot n_{j}=\left(h^{S} \sigma_{i j}^{S}(M)+H^{S} \sigma_{i j}^{S}(E)\right) \cdot n_{j} \quad ; \quad j=1,2$.

The equilibrium equation (A.12) may be regarded as a system of two scalar equations with three unknowns $\dot{\varepsilon}_{11}^{S}, \dot{c}_{1}$ and $\dot{c}_{2}$. By adding Eq. (A.1) to Eq. (A.12), we can formulate the resulting system, to be solved at each time increment $\left[t_{n}, t_{n+1}\right]$, in the following generic form:

$\mathbf{R}\left(\dot{\varepsilon}_{11}^{S}, \dot{c}_{1}, \dot{c}_{2}\right)=0$

where the components of the residual vector $\mathbf{R}$ are defined by

$\left\{\begin{array}{c}R_{1}\left(\dot{\varepsilon}_{11}^{S}, \dot{c}_{1}, \dot{c}_{2}\right)=(1+\rho) \dot{\varepsilon}_{11}^{S}+\dot{c}_{1} n_{1}+\dot{c}_{2} n_{2}-1=0 \\ R_{2}\left(\dot{\varepsilon}_{11}^{S}, \dot{c}_{1}, \dot{c}_{2}\right)=\left(h^{B} \sigma_{1 j}^{B}(M)+H^{B} \sigma_{1 j}^{B}(E)\right) n_{j}-\left(h^{S} \sigma_{1 j}^{S}(M)+H^{S} \sigma_{1 j}^{S}(E)\right) n_{j}=0 ; j=1,2 . \\ R_{3}\left(\dot{\varepsilon}_{11}^{S}, \dot{c}_{1}, \dot{c}_{2}\right)=\left(h^{B} \sigma_{2 j}^{B}(M)+H^{B} \sigma_{2 j}^{B}(E)\right) n_{j}-\left(h^{S} \sigma_{2 j}^{S}(M)+H^{S} \sigma_{2 j}^{S}(E)\right) n_{j}=0\end{array}\right.$

The above nonlinear system (A.14) should be solved iteratively (using the Newton-Raphson method, for example) in order to determine $\dot{\varepsilon}_{11}^{S}, \dot{c}_{1}$ and $\dot{c}_{2}$. Ultimately, the determination of these unknowns allows the computation of the different mechanical quantities at $t_{n+1}$. In particular, the two strain quantities $G_{33}^{S}\left(=\dot{\varepsilon}_{33}^{S}\right)$ and $\varepsilon_{11}^{S}$ are especially of interest; the first $G_{33}^{S}$ is required for the necking criterion (23) (in which the prescribed component $G_{33}^{B}$ is taken equal to -1 ), while the second $\varepsilon_{11}^{S}$ is needed in the algorithm of Section 4.

\section{Appendix B. Effect of the elastomer substrate on the ductility of the bilayer}

The aim of the current appendix is to explain, through some analytical developments, why the addition of an elastomer substrate leads to necking retardation for the resulting metal/elastomer bilayer. As a starting point of this development, let us consider the equilibrium Eq. (15). This equilibrium equation is equivalent to the following rate form:

$\overrightarrow{\mathbf{n}} .\left(h^{B} \dot{\mathbf{N}}^{B}(M)+H^{B} \dot{\mathbf{N}}^{B}(E)\right)=\overrightarrow{\mathbf{n}} \cdot\left(h^{S} \dot{\mathbf{N}}^{S}(M)+H^{S} \dot{\mathbf{N}}^{S}(E)\right)$,

where $\dot{\mathbf{N}}$ is the nominal stress rate related to the Cauchy stress rate $\dot{\boldsymbol{\sigma}}$ by the following relation:

$\dot{\mathbf{N}}=\dot{\boldsymbol{\sigma}}-\mathbf{G} . \boldsymbol{\sigma}$.

The unit vector $\overrightarrow{\mathbf{n}}$ normal to the necking band is restricted to rotate in the plane of the sheet. Hence, its third component $n_{3}$ is always equal to 0 . Therefore, the third components in Eq. (B.1) are equal to zero, and this equilibrium equation (B.1) can be reduced to its in-plane form. This in-plane form is considered in the remainder of this appendix. For the sake of simplicity and clarity, the same notations used in the general 3D case will be adopted again in the in-plane formulation. 
The nominal stress rate $\mathbf{N}$ is related to the velocity gradient $\mathbf{G}$ by the following generic form:

$\dot{\mathbf{N}}=\mathcal{L}: \mathbf{G}$,

where $\mathcal{L}$ is a tangent modulus which will be specified later.

By inserting Eq. (B.3) into Eq. (B.1), the equilibrium equation can be expressed in terms of $\mathbf{G}^{B}$ and $\mathbf{G}^{S}$

$\overrightarrow{\mathbf{n}} .\left(h^{B} \mathcal{L}^{B}(M)+H^{B} \mathcal{L}^{B}(E)\right): \mathbf{G}^{B}=\overrightarrow{\mathbf{n}} .\left(h^{S} \mathcal{L}^{S}(M)+H^{S} \mathcal{L}^{S}(E)\right): \mathbf{G}^{S}$,

which is equivalent to

$\overrightarrow{\mathbf{n}} \cdot\left(\mathcal{L}^{B}: \mathbf{G}^{B}\right)=\overrightarrow{\mathbf{n}} \cdot\left(\mathcal{L}^{S}: \mathbf{G}^{S}\right)$,

where $\mathcal{L}^{B}$ and $\mathcal{L}^{S}$ are defined by the following expressions:

$\mathcal{L}^{B}=h^{B} \mathcal{L}^{B}(M)+H^{B} \mathcal{L}^{B}(E) \quad ; \quad \mathcal{L}^{S}=h^{S} \mathcal{L}^{S}(M)+H^{S} \mathcal{L}^{S}(E)$.

The insertion of the compatibility condition (14) into the equilibrium equation (B.5) leads to the following equation:

$\left(\overrightarrow{\mathbf{n}} \cdot \mathcal{L}^{B} \cdot \overrightarrow{\mathbf{n}}\right) \cdot \overrightarrow{\mathbf{c}}=\overrightarrow{\mathbf{n}} \cdot\left(\left(\mathcal{L}^{S}-\mathcal{L}^{B}\right): \mathbf{G}^{S}\right)$,

which, when tensor $\overrightarrow{\mathbf{n}} \cdot \mathcal{L}^{B} \cdot \overrightarrow{\mathbf{n}}$ is invertible, is equivalent to

$\overrightarrow{\dot{\mathbf{c}}}=\left(\overrightarrow{\mathbf{n}} \cdot \mathcal{L}^{B} \cdot \overrightarrow{\mathbf{n}}\right)^{-1}\left(\overrightarrow{\mathbf{n}} \cdot\left(\left(\mathcal{L}^{S}-\mathcal{L}^{B}\right): \mathbf{G}^{S}\right)\right)$.

As defined by criterion (23), localized necking occurs when the velocity gradient in the band $\mathbf{G}^{B}$ becomes much larger, in norm, than its counterpart $\mathbf{G}^{S}$. Correspondingly, the jump vector $\overrightarrow{\dot{\mathbf{c}}}$ becomes unbounded at the onset of strain localization. A natural outcome from Eq. (B.8) is that the latter condition corresponds to the singularity of the acoustic tensor associated with the band $\overrightarrow{(\mathbf{n}} . \mathcal{L}^{B} . \overrightarrow{\mathbf{n}}_{\text {) }}$

$\|\overrightarrow{\dot{\mathbf{c}}}\| \rightarrow+\infty \Leftrightarrow \operatorname{det}\left(\overrightarrow{\mathbf{n}} \cdot \mathcal{L}^{B} \cdot \overrightarrow{\mathbf{n}}\right)=0$.

As previously given in Eq. (B.6), the tangent modulus in the band $\mathcal{L}^{B}$ is expressed as the weighted sum of two contributions: the first is related to the metal layer $\left(h^{B} \mathcal{L}^{B}(M)\right)$ and the second corresponds to the elastomer layer $\left(H^{B} \mathcal{L}^{B}(E)\right)$.

In the general case, and for both layers and zones, the tangent modulus $\mathcal{L}$ is defined by the following expression:

$\mathcal{L}=\mathbf{L}-\mathbf{L}_{1}-\mathbf{L}_{2}$,

where $\mathbf{L}$ is the instantaneous modulus relating the Jaumann co-rotational rate $\boldsymbol{\sigma}^{J}$ of the Cauchy stress tensor to the strain rate tensor $\dot{\boldsymbol{\varepsilon}}$ (the symmetric part of the velocity gradient $\mathbf{G}$ )

$\boldsymbol{\sigma}^{J}=\mathbf{L}: \dot{\boldsymbol{\varepsilon}}$.

As to $\mathbf{L}_{1}$ and $\mathbf{L}_{2}$, these are fourth-order tensors that convey the effect of convective stress components through the expression (B.11) of the tangent modulus $\mathcal{L}$. These tensors are given by the following general expressions:

$\forall i, j, k, l=1,2: \quad{ }_{1} L_{i j k l}=\frac{1}{2}\left(\sigma_{l j} \delta_{i k}+\sigma_{k j} \delta_{i l}\right) \quad ; \quad{ }_{2} L_{i j k l}=\frac{1}{2}\left(\sigma_{i k} \delta_{l j}-\sigma_{i l} \delta_{j k}\right)$.

Eq. (B.11) can be rewritten more explicitly in the following index form:

$\left\{\begin{array}{l}\sigma_{11}^{J}=L_{11} \dot{\varepsilon}_{11}+L_{12} \dot{\varepsilon}_{22} \\ \sigma_{22}^{J}=L_{12} \dot{\varepsilon}_{11}+L_{22} \dot{\varepsilon}_{22} \\ \sigma_{12}^{J}=2 L_{S} \dot{\varepsilon}_{12}\end{array}\right.$.

In what follows, the components $L_{11}, L_{12}, L_{22}$ and $L_{S}$ of the instantaneous moduli corresponding to each of the metal and elastomer layers will be given in detail.

1. Instantaneous modulus for the metal layer

Tvergaard [19] provided the analytical expressions for the components $L_{11}, L_{12}, L_{22}$ and $L_{S}$, in the case of incompressible and isotropic elastoplastic flow theory combined with mixed hardening

$$
\begin{cases}L_{11}=\frac{4}{3} E-\left(E-E_{T}\right)\left(\frac{\tilde{\sigma}_{11}}{\sigma_{Y}}\right)^{2} ; & L_{22}=\frac{4}{3} E-\left(E-E_{T}\right)\left(\frac{\tilde{\sigma}_{22}}{\sigma_{Y}}\right)^{2}, \\ L_{12}=\frac{2}{3} E-\left(E-E_{T}\right)\left(\frac{\tilde{\sigma}_{11} \tilde{\sigma}_{22}}{\sigma_{Y}^{2}}\right) ; \quad L_{S}=\frac{E}{3}\end{cases}
$$

where tensor $\tilde{\boldsymbol{\sigma}}$ is equal to $\boldsymbol{\sigma}-\mathbf{X}$, while $E$ and $E_{T}$ denote, respectively, the Young modulus and the scalar tangent modulus equal to $d \sigma_{e q} / d \varepsilon_{e q}$. The rigid-plastic behavior can be recovered as a limiting case of the elasto-plastic behavior, when $E \rightarrow \infty$.

2. Instantaneous modulus for the elastomer layer

The components of the instantaneous modulus corresponding to the neoHookean model are given by the following expression [7]: 
$\left\{\begin{array}{l}L_{11}=2 \mu\left(e^{2 \varepsilon_{11}}+e^{-2\left(\varepsilon_{11}+\varepsilon_{22}\right)}\right) \quad ; \quad L_{22}=2 \mu\left(e^{2 \varepsilon_{22}}+e^{-2\left(\varepsilon_{11}+\varepsilon_{22}\right)}\right) \\ L_{12}=2 \mu e^{-2\left(\varepsilon_{11}+\varepsilon_{22}\right)} ; \quad L_{S}=\frac{\mu}{2}\left(e^{2 \varepsilon_{11}}+e^{2 \varepsilon_{22}}\right)\end{array}\right.$

By analyzing Eqs. (B.14) and (B.15), we can easily observe that the tangent modulus of the elastomer layer remains constant or even increases slightly with stretching, while the tangent modulus of the metal layer decreases steadily. Therefore, compared to a freestanding metal layer at a given stretching level, the metal/elastomer bilayer has a stiffer tangent modulus, which promotes neck retardation. As a consequence, the addition of an elastomer substrate to a metal layer substantially enhances the ductility of the resulting bilayer.

\section{References}

[1] Chiu SL, Leu J, Ho PS. Fracture of metal-polymer line structures. I. Semiflexible polyimide. J Appl Phys 1994;76:5136-42.

[2] Hommel M, Kraft O. Deformation behavior of thin copper films on deformable substrates. Acta Mater 2001;49:3935-47.

[3] Alaca BE, Saif MTA, Sehitoglu H. On the interface debond at the edge of a thin film on a thick substrate. Acta Mater 2002;50:1197-209.

[4] Xue ZY, Hutchinson JW. Neck retardation and enhanced energy absorption in metal/elastomer bilayers. Mech Mater 2007;39:473-87.

[5] Xue ZY, Hutchinson JW. Neck development in metal/elastomer bilayers under dynamic stretchings. Int J Solids Struct 2008;45:3769-78.

[6] Jia Z, Li T. Necking limit of substrate-supported metal layers under biaxial in-plane loading. Int J Plast 2013;51:65-79.

[7] Ben Bettaieb M, Abed-Meraim F. Investigation of localized necking in substratesupported metal layers: comparison of bifurcation and imperfection analyses. Int J Plast 2015;65:168-90.

[8] Lacour SP, Jones J, Wagner S, Li T, Suo Z. Stretchable interconnects for elastic electronic surfaces. Proc IEEE 2005;93:1459-67.

[9] Cotton DPJ, Graz IM, Lacour SP. A multifunctional capacitive sensor for stretchable electronic skins. IEEE Sens J 2009;9:2008-9.

[10] Cordill MJ, Taylor A, Schalko J, Dehm G. Fracture and delamination of chromium thin films on polymer substrates. Metall Mater Trans A 2010;41A:870-5.

[11] Graudejus O, Jia Z, Li T, Wagner S. Size-dependent rupture strain of elastically stretchable metal conductors. Scr Mater 2012;66:919-22.

[12] Keeler SP, Backofen WA. Plastic instability and fracture in sheets stretched over rigid punches. Trans ASM, 56 1963; 1963. p. 25-48.

[13] Goodwin GM. Application of strain analysis to sheet metal forming problems in press shop. Metall Ital 1968;60(8):767-74.

[14] Stören S, Rice JR. Localized necking in thin sheets. J Mech Phys Solids $1975 ; 23: 421-41$.

[15] Rudnicki JW, Rice JR. Conditions for localization of deformation in pressuresensitive dilatant materials. J Mech Phys Solids 1975;23:371-94.

[16] Rice JR. The localization of plastic deformation. In: Proceedings of the 14th international congress of theoretical and applied mechanics; 1976. pp. 207-220.

[17] Marciniak Z, Kuczynski K. Limit strains in the processes of stretch-forming sheet metal. Int J Mech Sci 1967:9:609-20.

[18] Hutchinson JW, Neale KW. Sheet necking-III. Strain-rate effects. In: Koistinen DP, Wang NM, editors. Mechanics of sheet metal forming. New York: Plenum; 1978. p. 269-85.

[19] Tvergaard V. Effect of kinematic hardening on localized necking in biaxially stretched sheets. Int J Mech Sci 1978;20:651-8.

[20] Baudelet B. Prediction of forming limit diagrams for deep drawing. Agard Lect Ser 1984;137(3):1-22.

[21] Ghosh A. Tensile instability and necking in materials with strain hardening and strain-rate hardening. Acta Metall 1977;25:1413-24.

[22] Neale KW, Chater E. Limit strain predictions for strain-rate sensitive anisotropic sheets. Int J Mech Sci 1980;22:563-74.

[23] Cao J, Yao H, Karafillis A, Boyce MC. Prediction of localized thinning in sheet metal using a general anisotropic yield criterion. Int J Plast 2000;16:1105-29.

[24] Kuroda M, Tvergaard V. Forming limit diagrams for anisotropic metal sheets with different yield criteria. Int J Solids Struct 2000;37:5037-59.

[25] Haddag B, Abed-Meraim F, Balan T. Strain localization analysis using a large deformation anisotropic elastic-plastic model coupled with damage. Int J Plast 2009:25:1970-95.

[26] Abed-Meraim F, Balan T, Altmeyer G. Investigation and comparative analysis of plastic instability criteria: application to forming limit diagrams. Int J Adv Manuf Technol 2014;71:1247-62.

[27] Mansouri LZ, Chalal H, Abed-Meraim F. Ductility limit prediction using a GTN damage model coupled with localization bifurcation analysis. Mech Mater 2014;76:64-92.

[28] Hutchinson JW, Tvergaard V. Shear band formation in plane strain. Int J Solids Struct 1981:17:451-70.

[29] Chu CC. A three-dimensional model of anisotropic hardening in metals and its application to the analysis of sheet metal formability. J Mech Phys Solids 1984:32:197-212.

[30] Lu ZH, Lee D. Prediction of history-dependent forming limits by applying different hardening models. Int J Mech Sci 1987;29:123-37.

[31] Butuc MC, Teodosiu C, Barlat F, Gracio JJ. Analysis of sheet metal formability through isotropic and kinematic hardening models. Eur J Mech - A/Solids 2011;30:532-46.

[32] Tvergaard V. Effect of yield surface curvature and void nucleation on plastic flow localization. J Mech Phys Solids 1987:35:43-60.

[33] Armstrong PJ, Frederick CO. A mathematical representation of the multiaxial Bauschinger effect. Central Electricity generating Board Report, Berkeley Nuclear Laboratories, RD/B/N, 731; 1966.

[34] Hunter SC. Some exact solutions in the theory of finite elasticity for incompressible neo-hookean materials. Int J Mech Sci 1979;21:203-11.

[35] Rajagopal KR. On a class of elastodynamic motions in a neo-hookean elastic solid. Int J Non-Linear Mech 1998;33:397-405.

[36] Baghani M, Naghdabadi R, Arghavani J, Sohrabpour S. A thermodynamicallyconsistent $3 \mathrm{D}$ constitutive model for shape memory polymers. Int J Plast 2012;35:13-30.

[37] Bouvard JL, Francis DK, Tschopp MA, Marin EB, Bammann DJ, Horstemeyer MF. An internal state variable material model for predicting the time, thermomechanical, and stress state dependence of amorphous glassy polymers under large deformation. Int J Plast 2013;42:168-93.

[38] Uchida M, Tada N. Micro-, meso- to macroscopic modeling of deformation behavior of semi-crystalline polymer. Int J Plast 2013;49:164-84.

[39] Hutchinson JW, Neale KW, Needleman A. Sheet necking-I. Validity of plane stress assumptions of the long-wavelength approximation. In: Koistinen DP, . Wang NM, editors. Mechanics of sheet metal forming. New York: Plenum; 1978. p. 111-26.

[40] 3DS Report. Selection and identification of elastoplastic models for the materials used in the benchmarks. 18-Months Progress Report, International IMS Research Contract Digital Die Design Systems (3DS). Laboratory of the Mechanical and Thermodynamic Properties of the Materials, University Paris 13, Villetaneuse, France; 2001

[41] Graf AF, Hosford WF. Calculations of forming limit diagrams for changing strain paths. Metall Trans A 1993;24a:2497-501.

[42] Graf AF, Hosford WF. Effect of changing strain paths on forming limit diagram of Al 2008-T4. Metall Trans A 1993;24a:2503-12.

[43] Logan R, Hosford WF. Upper-bound anisotropic yield locus calculations assuming (111)-pencil glide. Int J Mech Sci 1980;22:419-30.

[44] Nurcheshmeh M, Green DE. Prediction of sheet forming limits with Marciniak and Kuczynski analysis using combined isotropic-nonlinear kinematic hardening. Int J Mech Sci 2011;53:145-53.

[45] Yao H, Cao J. Prediction of forming limit curves using an anisotropic yield function with prestrain induced backstress. Int J Plast 2002;18:1013-38.

[46] Amirkhizi AV, Isaacs J, McGee J, Nemat-Nasser S. An experimentally-based viscoelastic constitutive model for polyurea including pressure and temperature effects. Philos Mag 2006;86:5847-66. 